\title{
Medievalista
}

Online

$26 \mid 2019$

Número 26

\section{Etudes menées sur les sculptures d'Auvergne en bois polychromé}

Polychrome wood sculptures in Auvergne, an ever-evolving corpus: state of the research and report of technical analysis

Marie-Blanche Potte, Dominique Faunières, Agnès Blossier et Lucretia Kargère

\section{OpenEdition}

\section{Journals}

Édition électronique

URL : http://journals.openedition.org/medievalista/2333

DOI : 10.4000/medievalista.2333

ISSN : 1646-740X

Éditeur

Instituto de Estudos Medievais - FCSH-UNL

Édition imprimée

Date de publication : 1 juin 2019

Référence électronique

Marie-Blanche Potte, Dominique Faunières, Agnès Blossier et Lucretia Kargère, «Etudes menées sur les sculptures d'Auvergne en bois polychromé », Medievalista [En ligne], 26 | 2019, mis en ligne le 18 juin 2020, consulté le 10 décembre 2020. URL : http://journals.openedition.org/medievalista/2333 ; DOI : https://doi.org/10.4000/medievalista.2333

\section{(c) (1) 8}

Mediavalista está licenciado com uma Licença Creative Commons - Atribuição-NãoComercial 4.0 Internacional. 
Título / Title: Etudes menées sur les sculptures d'Auvergne en bois polychromé / Polychrome wood sculptures in Auvergne, an ever-evolving corpus: state of the research and report of technical analysis

Autor(es) / Author(s):

Marie-Blanche Potte (a); Dominique Faunières (b); Agnès Blossier (c); Lucretia Kargère (d) Afiliação institucional (Unidade de Investigação, Faculdade ou Departamento, Universidade, Código postal, Cidade, País) / Institutional Affiliation (Research Center, Faculty or Department, University, Postcode, City, Country):

(a) Conservatrice en chef du patrimoine, Lyon, France; (b) Restauratrice indépendante de sculptures, Paris, France; (c) Restauratrice indépendante de sculptures, Tours, France;

(d) Restauratrice du Cloisters, The Metropolitan Museum of Art, New York, USA Email Institucional / Institutional email:

(a) marie-blanche.potte@culture.gouv.fr; (b) domfo@wanadoo.fr;

(c) agnès.blossier@gmail.com; (d) lucretia.kargere@metmuseum.org

Fonte: Medievalista [Em linha]. Direc. Bernardo Vasconcelos e Sousa. Lisboa: IEM.

Disponível em:

http://www2.fcsh.unl.pt/iem/medievalista/MEDIEVALISTA26/potte-et-al2608.html ISSN: 1646-740X

DOI: $10.4000 /$ medievalista.2333

Data recepção do artigo / Received for publication: 20 de novembro de 2018

Data aceitação do artigo / Accepted in revised form: 29 de abril de 2019 


\section{Abstract}

In France, the Auvergne region has preserved an impressive number of polychrome wood sculptures. For those interpreted as dated between the $11^{\text {th }}$ and $13^{\text {th }}$ centuries, there are about 20 figures of the Crucifix, over 60 groups of the Virgin and Child in Majesty, and rarer saint figures, not including wooden sculptures sheeved in metal. While key prototypes held in museum collections have acquired a certain prestige, a whole corpus displayed in regional churches remains unknown to the public, little examined on a technical level. Since 2010, following a noticable shift of interest for these sculptures, the DRAC Auvergne-Rhône-Alpes has undertaken to define a corpus, and to direct a technical study of select sculptures. As cult figures, these objects have experienced continuous changes over centuries. The study proposes to contextualize the sculptures in their original church environment. The in-depth examination of a group of monumental crucifixes showing remarkable similarities of materials and techniques, asks for a reevaluation of the working methods of early medieval sculptors and painters, with the possible use of models. The examination of groups of the Virgin and Child echoes this possibility of a standardized production, although noted differences between the sculptures put in question the concept of serial production.

Keywords: Auvergne, Sculpture, Carving, Painting technique, Models.

\section{Résumé}

En France, la région d'Auvergne conserve un important ensemble de sculptures en bois polychromées datées, pour le moment entre le 11eme et le début du 13eme siècle, notamment une vingtaine de Crucifix, une soixantaine de Vierge en majesté et diverses figures de saints. Tandis que les sculptures conservées dans les musées ont acquis un certain prestige, celles qui sont conservées dans leurs églises restent moins connues et ont rarement fait l'objet d'études techniques. Depuis 2010 et l'émergence d'un nouvel intérêt pour ces objets, la DRAC ARA entreprend l'inventaire du corpus et initie de nouvelles recherches. En tant qu'objets de cultes, ces sculptures ont subi de nombreuses 
transformations au cours des siècles. Cette étude propose de contextualiser la place de ces objets dans leur édifice. L'examen d'un groupe de crucifix monumentaux montre la remarquable similitude d'élaboration originelle, nous incitant à nous interroger sur la production de modèles dans les ateliers de sculpteurs et de polychromeurs, et à re-évaluer notre vision des modes de production. L'étude des Vierges en majesté fait également écho à cette question du processus artistique et d'imitation de prototypes entiers, tout en marquant les variations qui définissent chacune de ces oeuvres.

Mots clefs: Auvergne, Sculpture, Bois, Polychromie, Modèles. 


\title{
.
}

\section{Etudes menées sur les sculptures d'Auvergne en bois polychromé / Polychrome wood sculptures in Auvergne, an ever-evolving corpus: state of the research and report of technical analysis}

\author{
Marie-Blanche Potte, Dominique Faunières, Agnès Blossier, Lucretia Kargère
}

En France, l'un des traits les plus intéressants de la région d'Auvergne est le pourcentage de sculptures religieuses en bois polychromé supérieur à la moyenne nationale. Pour les sculptures interprétées comme datant du $11 \mathrm{eme}$ à la première moitié du $13 \mathrm{eme}$ siècle, on dénombre une vingtaine de Crucifix, plus de soixante Vierges en majesté, et de rares sculptures représentant un saint, sans compter celles composées d'une âme de bois recouverte de lames de métal. En Auvergne leur nombre reste nécessairement variable car beaucoup de questions demeurent en suspens quant à leur date. Des exemples peuvent être considérés comme des œuvres tardives avec des aspects "romanisants", d'autres comme des copies plus tardives fidèles au canon roman. Beaucoup de ces sculptures sont conservées à l'abri soit dans des chapelles dans une région restée extrêmement rurale, soit préservées dans des musées en France, ou même à l'étranger. Bien que les Vierges en majesté aient fait l'objet de plus amples recherches stylistiques, ce corpus est finalement assez mal compris, car mal étudié d'un point de vue scientifique ${ }^{1}$. Comme le démontre la

\footnotetext{
${ }^{1}$ ALEIL, Pierre François - "Les grands Crucifix de bois en Haute-Auvergne", Revue de la Haute-Auvergne 38 (1963), pp.219-36; FOUILHERON, Joël - "Du bon Dieu de Saint-Flour au Christ noir de la Cathédrale", Revue de la Haute-Auvergne 39 (1965), pp.490-96; FORSYTH, Ilene H - The Throne of Wisdom: Wood Sculptures of the Madonna in Romanesque France, 1972; BOUSQUET, Jacques - "Sur quelques Christ romans du Rouergue et le problème des "christs Auvergnats", Actes du Congrès d'études de Rodez (1975), pp. 333-359; LITTLE, Charles - "Romanesque sculpture in North American collections: The Metropolitan Museum of Art; Part VI: Auvergne, Burgundy, Central France, Meuse Valley, Germany”, Gesta 26/2 (1987); DELMAS, Claire - "Le Crucifix Roman de Salles-la-Source: Nouveaux éléments concernant les grands Christs en bois du Massif Central", Revue de la Haute-Auvergne 9 (1990), pp.49-54; CARRIÈRE, Adeline. "Les grands crucifix romans de bois de l'Auvergne et de ses marges", Recherche en histoire de l'art (2008), pp.7-28; LEROY, Hélène et DEBAISIEUX, Francis - Vierges romanes, portraits croisés, Beaumont: Edition Debaisieux, 2009; BOEHM, Barbara - "Un buste reliquaire du 12ème siècle
} 
série d'articles dans ce numéro, le manque d'information est plus ou moins généralisé pour l'Europe, car l'étude des sculptures en bois polychromé a largement été mise de côté vu la difficulté d'étudier ces objets, souvent abîmés par le temps et l'histoire, modifiés par les progressions de leur culte, avec peu de suivi en archives. Rares sont les sculptures restées dans leur emplacement d'origine et de nombreuses questions subsistent quant au contexte de leur création: le nombre d'ateliers régionaux, les possibles commanditaires, l'aspect même de ces sculptures à l'origine, ainsi que leur date d'exécution.

Depuis 2010, l'étude détaillée des sculptures en bois polychromé d'Auvergne connait un net essor, le fruit d'un programme de recherche interdisciplinaire mené par la DRAC, d'études scientifiques de certaines sculptures in situ ou en laboratoire, et d'examens entrepris au Cloisters, la branche médiévale du Metropolitan Museum de New York ${ }^{2}$. Cet article est un compte rendu du programme complété, notamment de l'étude scientifique d'un nombre de Christs en croix jamais diffusé jusqu'ici.

\section{Marie-Blanche Potte:}

Fédérer, orienter et diffuser les études menées sur les sculptures en bois polychromé d'Auvergne

En DRAC Auvergne-Rhône Alpes, le corpus très important des sculptures médiévales de bois polychromé restées dans leur édifice d'origine nous a poussé à structurer la recherche à l'échelle de toute la région, avec l'objectif de diffuser et faire connaître les œuvres et l'actualité des recherches et travaux qui les concernent. L'organisation du Ministère de la culture en France, à travers les Directions régionales des affaires culturelles (DRAC), lui permet d'être présent sur tout le territoire. Dans les DRAC, les conservations régionales des monuments historiques assurent le suivi des immeubles et des œuvres non gardées,

récemment découvert à Saint-Flour", Patrimoine en Haute Auvergne 23/2 (2011), pp. 39-50; GIRELLI, Francesca - "Local traditions and itinerant artists?: The Crucifixes of Auvergne and Wooden Sculpture in France during the Late Romanesque Period", Convivium, Exchanges and Interactions in the Arts of Medieval Europe, Byzantium, and the Mediterranean IV (2017), pp. 53-70; MOULIER, Pierre. Christs romans d'Auvergne, Patrimoine en Haute Auvergne 35, 2018.

2 KARGĖRE, Lucretia; RIZZO, Adriana - "Twelfth-century French polychrome sculpture in The Metropolitan Museum of Art: materials and techniques", Metropolitan Museum Studies in Art, Science and Technology 1 (2010), pp. 39-72. 
classées ou inscrites au titre des monuments historiques, présentes dans toutes les communes de France. Exerçant repérage, protection, suivi des restaurations et des travaux, les conservateurs des monuments historiques ont accès à un corpus d'œuvres souvent méconnu, plus vaste que celui des musées, et sur lesquels restaurateurs et chercheurs travaillent au quotidien.

\section{Le corpus}

Souvent réduit aux "Vierges auvergnates", le corpus régional de sculpture médiévale polychromée est en réalité plus large. A l'image des Vierges, les œuvres en bois médiévales sont en Auvergne-Rhône-Alpes extrêmement nombreuses, identifiées pour certaines de longue date, moins connues pour d'autres, et forment un corpus identitaire fort. Pourtant, les nombreuses études menées à l'occasion de restaurations récentes restent consignées dans les services sans que leur réunion, leur diffusion, leur synthèse, leur présentation à la fois au public scientifique et au grand public ne soit projetée.

Les récentes études menées sur des patrimoines comparables montrent une évolution rapide de l'historiographie, qui nuance notamment les datations et les limites chronologiques jusqu'ici reconduites ${ }^{3}$. Lors des restaurations menées ces dernières années, les Christs médiévaux, notamment, ont laissé apparaître une permanence des modèles dont la datation s'avère malaisée, mais qui possèdent des qualités de sculpture et de polychromie remarquables.

\section{Recensement des études existantes}

Le premier travail de cette étude, permettant la définition des problématiques majeures, a consisté en un recensement des études existantes, et des restaurations récentes ayant donné lieu à un rapport complet archivé en DRAC. Entre 2010 et 2018, de nombreuses œuvres ont fait l'objet d'études, parfois partielles, mais dont les rapports très complets ont permis de mettre en place les grandes questions restant centrales dans l'étude de ce

\footnotetext{
${ }^{3}$ DESCHAMPS-TAN, Stephanie et al. - "La Vierge et l'Enfant en majesté, musée du Louvre", Technè 39 (2014), pp. 66-72; CASCIO, Agnès et al. "La restauration du Christ Courajod: la luminosité d'une polychromie romane retrouvée", Technè 39 (2014), pp.52-59; BERTONI CREN, Nadia - La sculpture sur bois polychrome des $11^{\mathrm{e}}$ et $12^{\mathrm{e}}$ siècles en Bourgogne, Université de Bourgogne, PhD Thesis, 2013.
}

Medievalista № 26 | Julho - Dezembro 2019 ๑ IEM - Instituto de Estudos Medievais 6 www2.fcsh.unl.pt/iem/medievalista 
corpus. Une première liste a été établie, des œuvres étudiées dans les dix dernières années, et des œuvres identifiées mais n'ayant pas fait l'objet d'une actualisation de la connaissance.

Conjointement au corpus recensé et étudié en Auvergne, un certain nombre d'œuvres “déplacées" ont pu être identifiées; elles ont donné lieu à des dialogues qui viennent enrichir l'approche: c'est le cas notamment des deux Christs provenant d'Auvergne du Musée National du Moyen-Age, de la Vierge en majesté du Louvre, des deux Vierges auvergnates du Metropolitan Museum de New-York, du Christ de Lavaudieu dans ce même musée ${ }^{4}$.

Ces œuvres, publiées et étudiées, permettent d'appréhender des modes de description et d'étude différents, ouvrant sur la recherche internationale, et d'interroger celles à mettre en place aux côtés du corpus auvergnat. Elles désignent, par ailleurs, des interlocuteurs internationaux susceptibles de dialoguer autour de notre corpus.

\section{État des questions émergentes}

A la lecture des rapports de restauration de ces dix dernières années, émergent trois problématiques centrales dans la connaissance des œuvres de ce corpus.

Premièrement, la stratigraphie colorée. Les œuvres médiévales, souvent très complexes dans leur stratigraphie colorée, sont infiniment riches d'enseignement sur l'histoire, les dévotions, et le territoire étendu de la réunion physique des matériaux (pigments notamment). En premier lieu, l'étude des polychromies d'origine au laboratoire fournit des données essentielles pour la connaissance de l'œuvre dans son premier état. Par la suite, l'analyse des états intermédiaires enrichit amplement la connaissance de l'évolution de ces sculptures, où l'état d'origine est souvent très lacunaire. Le dégagement, par

\footnotetext{
${ }^{4}$ BERNÉ, Damien - "Le Christ de Crucifixion de la collégiale d'Herment (Puy-de-Dôme), Une redécouverte exceptionnelle au musée de Cluny", Fines, Archéologie et Histoire en Combraille 6 (2011), pp. 17-25; GABORIT, Jean-René et FAUNIĖRES, Dominique - Une Vierge en majesté, Musée du Louvre Editions, 2009; KARGĖRE, Lucretia; RIZZO, Adriana - "Twelfth-century French polychrome sculpture...", pp. 39-72; KARGÈRE et al. - "Un Christ roman auvergnat retrouve son unité grâce à l'étude de la polychromie", TECHNE 39 (2014), pp. 60-65.
} 
exemple, sur la Vierge en majesté romane de Courpière, de la robe du 16ème siècle encore présente sous la polychromie 19ème, donne à voir une dévotion dans son temps et un territoire perméable aux modes et aux échanges ${ }^{5}$.

La question des Vierges ou Christs noirs, dont le nombre est particulièrement important en Auvergne, ne peut trouver de réponses que grâce aux études scientifiques des couches de stratigraphie ${ }^{6}$. Les premiers éléments d'études récentes ont démontré la complexité de la question: si le noircissement du corps du Christ de Saint-Flour semble être survenu relativement tardivement (première moitié du 19ème siècle), en revanche, les sondages opérés en 2017 sur le Christ de Montsalvy font apparaître assez tôt la présence d'une épaisse couche noire, située sur le premier repeint des carnations et au-dessous de sept autres repeints. L'analyse de cette couche conclut bien à une véritable application noirâtre, chargée de noir d'os et de pigments silicatés verdâtres, sans doute d'aspect assez translucide. Ce noircissement ancien, repéré sur d'autres œuvres romanes ou tout début gothique, notamment sur la Vierge de Montvianeix du musée du Cloisters à New York comme $1^{\text {er }}$ repeint, montre qu'une réponse simpliste de diffusion d'un seul modèle limité dans le temps n'est pas suffisante, et que le corpus doit être augmenté pour comprendre une pratique élargie historiquement ${ }^{7}$.

En second lieu, la présence de caches à reliques dans la statuaire, mais aussi d'œuvres repercées dans le temps, réemployées, "rechargées", prennent une place particulière. Jusqu'ici, les moyens d'investigation n'avaient pas permis une connaissance fine - et non destructive - des œuvres sur ce point. Le recours à l'imagerie récente (scanner, notamment) a fait considérablement évoluer la question, et a permis de belles découvertes, non seulement dans les Vierges en majesté dont la présence de caches à relique a déjà été diffusé, mais aussi dans les christs monumentaux ${ }^{8}$.

\footnotetext{
${ }^{5}$ Rapport de restauration de la Vierge en majesté de Courpière, Juliette Levy, 2015, non publié, archives de la DRAC ARA.

${ }^{6}$ CASSAGNES-BROUQUET, Sophie - Vierges Noires, Rodez: ed. du Rouergue, 2000.

${ }^{7}$ BRÉHIER, Louis - "Notes sur les Statues de Vierges Romanes, Notre Dame de Montvianeix et la question des Vierges Noires", Revue d'Auvergne 47 (1933), pp.193-198; KARGÈRE, Lucretia - "The Montvianeix Madonna: materials and techniques in $12^{\text {th }}$-century Auvergne”, Proceedings ICOM Committee for Conservation 13 ${ }^{\text {th }}$ Triennial Meeting Rio de Janeiro, vol. 2 (2002), p. 508.

${ }^{8}$ KARGÈRE et RIZZO -“Twelfth-century French polychrome sculpture...”, pp. 48-49; FAUNIÈRES et BLOSSIER, rapport d'étude du Christ de Saint-Flour, 2017.
} 
Enfin, comme troisième axe de recherche, les rares travaux ayant exploré le placement de l'œuvre dans l'édifice ont démontré que la connaissance était encore balbutiante sur ce point $^{9}$. Des traces matérielles de celle-ci ont été documentées sur le bâti comme à Orcival, ou bien encore sur l'œuvre, comme à Brioude ${ }^{10}$. Se peut-il notamment que l'échelle et l'envergure des sculptures, qui par ailleurs présentent de grandes ressemblances, aient été liées au lieu d'emplacement d'origine? ${ }^{11}$ Accompagner la restauration d'une étude historique sur le placement de l'œuvre, c'est permettre, au retour de restauration, une mise en place raisonnée de la sculpture, plutôt qu'une mise sous cloche guidée par une culture plus muséale que monumentale. Ce travail de réflexion aborde la statuaire de bois médiévale comme un objet architectural, à la frontière entre l'œuvre isolée et l'élément d'indexation liturgique de l'église. La mutation des architectures qui hébergent aujourd'hui les œuvres interdit souvent de revenir à un placement "originel"; cependant, une meilleure connaissance de ce placement - poutres de gloire, images réputées acheiropoïètes, etc. - peut enrichir considérablement l'accompagnement de ces œuvres.

\section{Dominique Faunières et Agnès Blossier: \\ Étude de deux Christs romans auvergnats - hypothèse d'une production en série}

En 2016 une première étude technologique est lancée par la Direction Régionale des Affaires Culturelles de la Région Auvergne-Rhône-Alpes sur le Christ noir de Saint-Flour (Cantal), pour lequel l'attribution au 15ème siècle est discutée depuis son arrêté de classement en 1908. Puis en 2017 une seconde étude est engagée sur le Christ de Montsalvy (Cantal), œuvre polychromée, moins connue, également datée du 15ème siècle lors de son arrêté de classement en 1902 (fig. 1).

\footnotetext{
${ }^{9}$ Voir notamment PLAGNIEUX, Philippe -“D'une chapelle de la Vierge l'autre: l'exemple du prieuré clunisien de Saint-Martin-des-Champs à Paris", Bulletin du centre d'étude médiévales d'Auxerre, BUCEMA, Hors-série 6 (2013), pp.5-6.

${ }^{10}$ MOREL, D. - Notre-Dame d'Orcival, Auvergne, Service régional de l'inventaire général du patrimoine culturel; POTTE, Marie-Blanche (dir.), Lyon, Lieux Dits, 2008.

${ }^{11}$ FACHECHI, Maria-Grazia - "Forma quantitate et qualitate diligentissime denotata la scultura lignea medievale tra spazio e funzione", Annali della pontificia insigne accademia di belle arti lettere dei virtuosi al Pantheon (2015), pp. 377-388.
} 

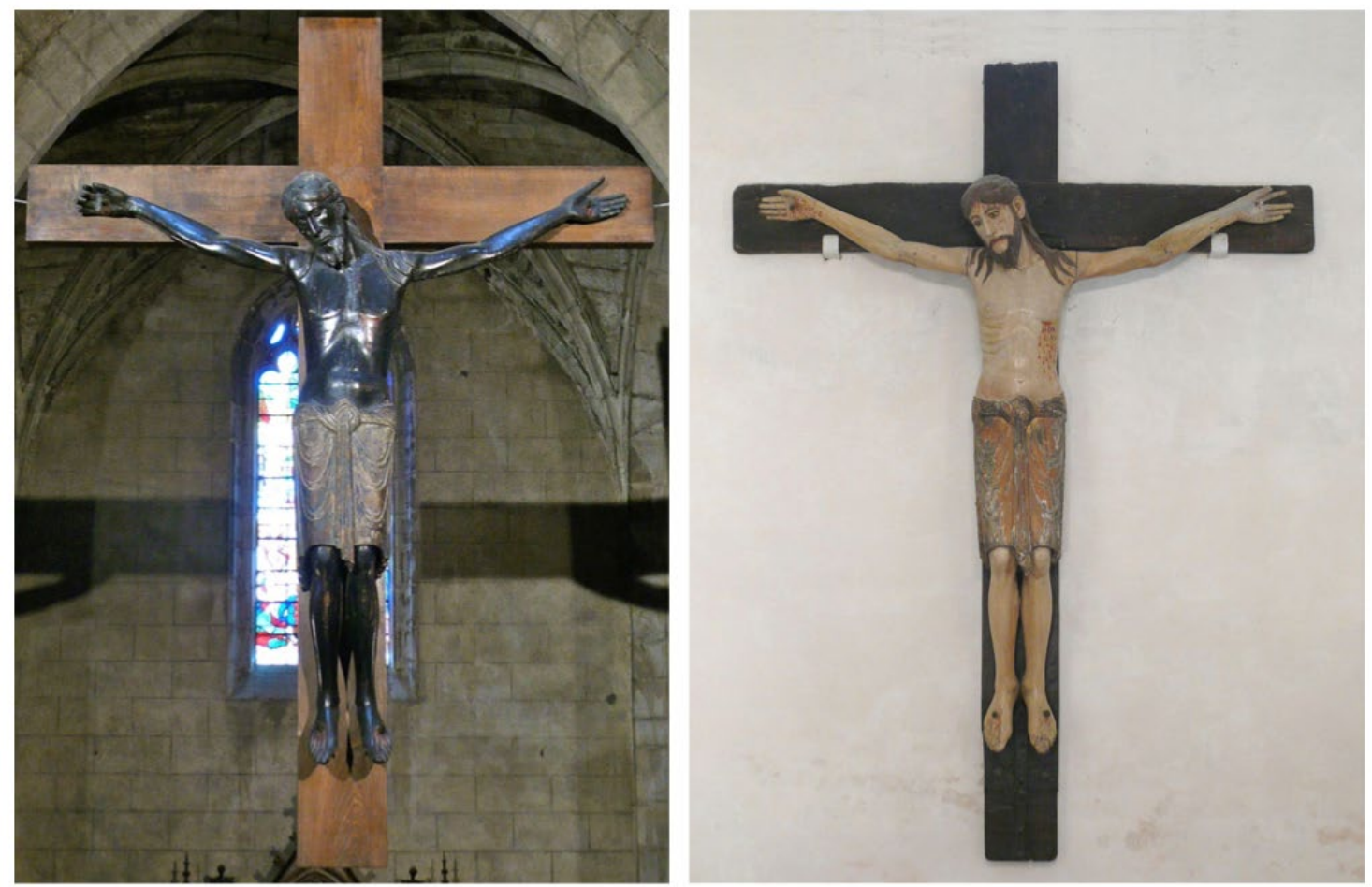

Fig. 1 - Le Christ de Saint-Flour, bois polychromé, 12ème-13ème siècle, conservé à la cathédrale Saint-Pierre (à gauche) et le Christ de Montsalvy, bois polychromé, 12ème-13ème siècle, conservé à l'abbatiale Notre-Dame-de-l'Assomption (à droite).

(C) Dominique Faunières et Agnès Blossier)

Chacune des études ${ }^{12}$ a été réalisée in situ après la dépose des Crucifix. Seul le Christ de Saint-Flour a pu être déplacé pour être scanné tandis que le Christ de Montsalvy a bénéficié d'une radiographie ambulatoire. Des datations au carbone $14^{13}$ ont été réalisées dans les deux cas, ainsi que des analyses pour l'identification des essences de bois ${ }^{14}$ et des analyses des polychromies ${ }^{15}$. À notre sens ces deux Christs sont identiques tant du point de vue stylistique que technologique, à quelques détails de mise en œuvre près. La récurrence de certains dispositifs techniques encore en place ou sous forme de traces, a permis de conclure à l'originalité de ceux-ci. La croix du Christ de Saint-Flour est très récente, alors que celle de Montsalvy est authentique - le Christ de Montsalvy n'a jamais

\footnotetext{
${ }^{12}$ Les observations ont été réalisées à l'œil nu, aux lunettes loupe et à la loupe binoculaire à grossissement variable (optique Zeiss). Emploi d'une micro caméra numérique.

13 Tomasz Goslar, responsable du Poznan Radiocarbon Laboratory, Pologne.

${ }^{14}$ Emmanuel Maurin, Laboratoire de Recherche des Monuments Historiques, Champs-sur Marne.

${ }^{15}$ Marie-Pierre Etcheverry, laboratoire LAMOA, Bordeaux.
} 
été déposé de sa croix. Par ailleurs, dès la première étude, il est apparu pertinent de rapprocher ces deux Christs monumentaux d'un troisième Christ conservé dans l'église du prieuré de Lavoûte-Chilhac (Haute-Loire). La mise en parallèle des caractéristiques de cette œuvre avec les deux Christs est utilisée et développée dans notre exposé.

\section{Des éléments iconographiques et stylistiques similaires}

Les deux Christs appartiennent à un groupe de Christs en croix monumentaux dont les caractéristiques de base sont communes à bien des crucifix de l'Auvergne, comme audelà. Leurs traits communs sont les suivants: le Christ possède une tête non couronnée, légèrement penchée en avant et sur son côté droit à la chevelure épaisse ramenée derrière de larges oreilles. Il est doté d'un périzonium arrivant juste au-dessus des genoux. Pour autant les Christs étudiés ici semblent appartenir selon nous à un sous-groupe présentant des particularités de traitement iconographique et stylistique. Au cours des siècles, le parcours individuel des Christs étudiés a plus ou moins fortement modifié leur apparence, en particulier leur aspect coloré: le Christ de Saint-Flour est devenu tardivement un Christ noir connu sous l'appellation de "Bon Dieu noir", tandis que celui de Montsalvy a été progressivement empâté par de nombreux repeints. Ils ont également subi des transformations formelles de parties du corps et de la croix, telles que les mains, les épaules et une partie du côté dextre du périzonium du Christ de Saint-Flour, et de la croix. L'analyse est donc malaisée à première vue mais le rapprochement des détails de la sculpture demeure significatif. L'expression du visage est calme, les yeux sont ouverts mais leur fente est relativement étroite, le nez - à l'arête large et aux narines plaquées est long et droit, la bouche est fine aux commissures tombantes (fig. 2). Les oreilles sont de grande taille et détaillées. Les bras sont très légèrement levés à l'oblique. La chevelure est sculptée sans discontinuité sur l'arrière et le dessus de la tête, confirmant qu'à l'origine le Christ ne portait pas de couronne. Trois mèches sculptées retombent sur chaque épaule $^{16}$. L'agencement particulier des lourdes mèches de cheveux se retrouve à l'identique sur les deux Christs (fig. 3). Il en va de même de l'organisation des touffes de poils sur les tempes et de celle des boucles de la barbe, ainsi que de l'orientation légèrement oblique et de la facture schématique de la moustache. Au dos, les mèches de

\footnotetext{
${ }^{16}$ La restauration effectuée sur le Christ de Saint-Flour par l'atelier André entre 1946-1947 a modifié certains volumes, notamment en supprimant deux mèches sur l'épaule droite.
} 
la chevelure s'arrêtent au bas de la nuque qui correspond au joint d'assemblage de la tête. La chevelure, la barbe et la moustache sont représentées de manière stylisée. Les cheveux regroupés en mèches, globalement parallèles sur le dessus de la tête, ondulent en vagues dont les sommets sont alternés d'une mèche à l'autre. Les mèches et les boucles de la barbe et de la chevelure sont placées très précisément (fig. 4). Chaque volume est ensuite ciselé de fines lignes parallèles, caractérisées par une crête plate ${ }^{17}$.
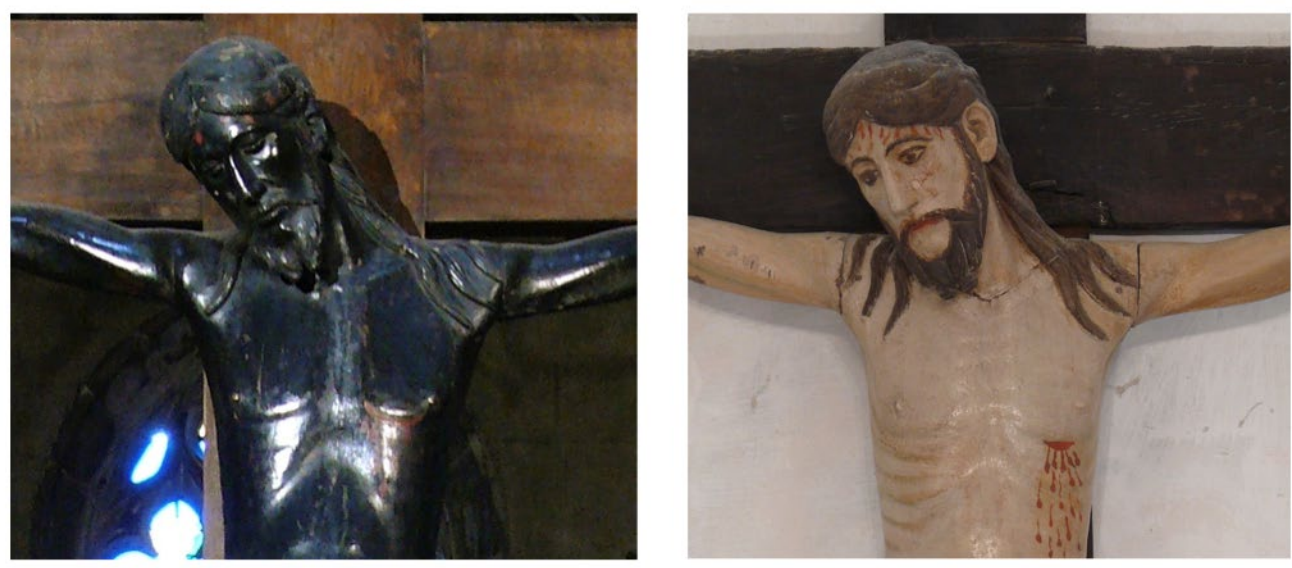

Fig. 2 - Les têtes des Christ de Saint-Flour et de Montsalvy. (C) Dominique Faunières et Agnès Blossier)
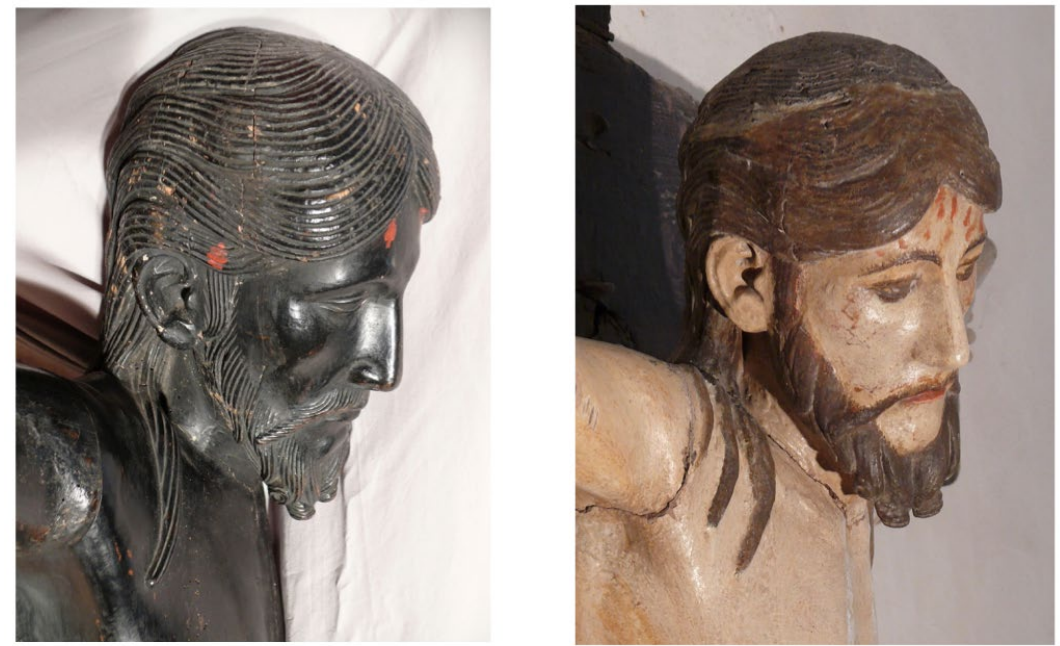

Fig. 3 - Détails de la chevelure des Christ de Saint-Flour et de Montsalvy.

(C) Dominique Faunières et Agnès Blossier)

\footnotetext{
${ }^{17}$ Lors de l'étude du Christ de Saint-Flour nous nous sommes demandées si cette caractéristique résultait du décapage du bois avant noircissement, mais sa présence sur le Christ de Montsalvy et celui de LavoûteChilhac tend à conclure que c'est une facture originelle commune.
} 

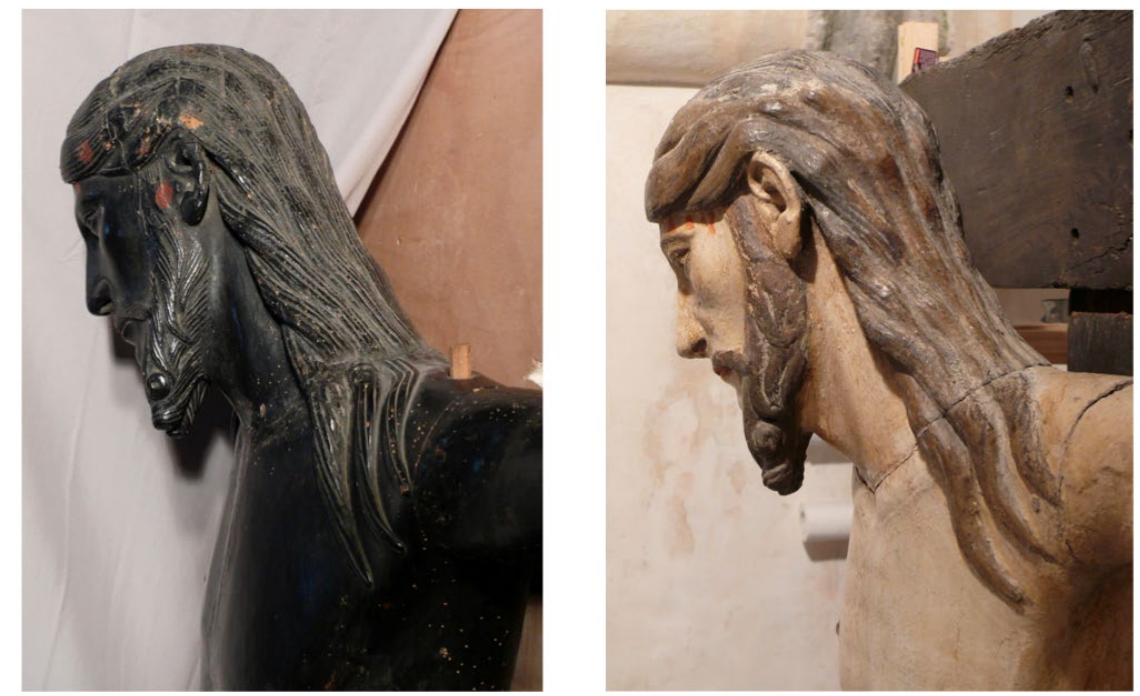

Fig. 4 - Détails de la barbe et des tempes des Christ de Saint-Flour et de Montsalvy.

(C) Dominique Faunières et Agnès Blossier)

Le modelé de la cage thoracique est doux. Les côtes sont peu marquées et le sternum n'est pas détaillé. Le nombril et la pointe des mamelons sont sculptés mais pas la plaie sur le côté. Le revers des bras est sculpté, ce qui se comprend car le torse est légèrement porté en avant et la forme des omoplates est esquissée. La musculature des bras forme une torsade, celle des avant-bras reste peu détaillée. Malgré la réfection de deux doigts, les mains du Christ de Montsalvy témoignent de la conception d'origine, celles du Christ de Saint-Flour sont des réfections d'époques différentes. Les mains originelles sont proportionnées au reste du corps.

Le périzonium est composé de chaque côté de quatre plis droits, tombant verticalement, tandis que sur chacune des cuisses fléchies, le drapé plus tendu, séparé par la retombée du nœud, forme une série de trois doubles plis en arcs de cercle croisés, espacés et relativement peu saillants (fig. 5). Un quatrième double pli est à peine esquissé en partie haute. L'agencement complexe du tissu sur les hanches, des deux côtés du nœud, ne suit apparemment pas de logique de drapement. Ainsi les plis de la "ceinture" en forme de "chevrons" de part et d'autre de l'arrière des hanches, se poursuivent en bandeaux pour former au centre un nœud par lequel glisse un seul pan vertical, étagé en trois retournements (fig. 5) ${ }^{18}$.

\footnotetext{
${ }^{18}$ Notons que l'agencement en "chevrons" et le plissé du pan central se retrouvent sur d'autres Christs auvergnats et rouergats tels ceux de Blesle, Thérondels, Salles-la-Source, etc...
} 

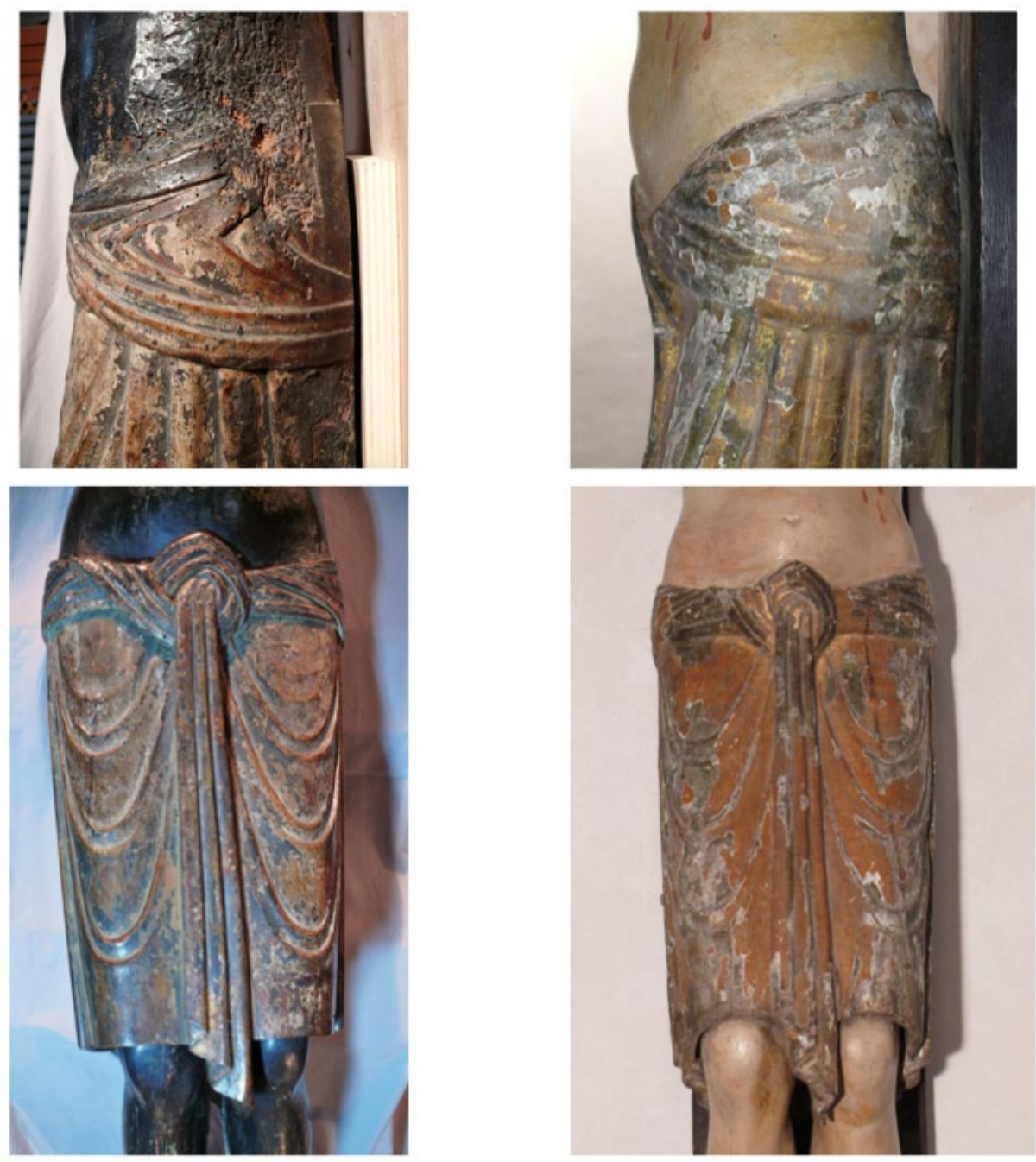

Fig. 5 - Périzonium des Christ de Saint-Flour et de Montsalvy.

(C) Dominique Faunières et Agnès Blossier)

Les jambes sont mises en forme même pour les parties cachées par le périzonium. La plante des pieds est modelée; elle doit en effet avoir été visible car il n’y avait pas de suppedaneum à l'origine comme l'atteste l'absence d'implantation sur la croix de Montsalvy.

Le Christ est fixé à sa croix par quatre clous; le rôle de soutien est assuré par un système placé originellement au revers du bassin. Les affranchissements des extrémités de la croix originale de Montsalvy ne permettent pas de connaître sa forme globale. Les largeurs de la traverse et du montant ne vont pas au-delà de la forme du corps. 


\section{La mise en œuvre originale}

Les Christs de Saint-Flour et de Montsalvy ont été taillés dans du noyer. Le bloc initial du torse et du périzonium comprenait le cœur de l'arbre. Les images scanner du Christ de Saint-Flour permettent de visualiser l'emplacement du cœur de la bille de bois à l'intérieur du torse, ainsi nous comprenons que le bois employé pour celui-ci était tors. Sur le plan de joint des épaules, le cœur est centré puis il dévie vers la hanche droite et la surface de la sculpture. Ce dernier trait, les défauts révélés par le scanner, ainsi que la présence du cœur de l'arbre, montrent que ce n'est pas la qualité technique qui a prévalu dans la sélection de l'arbre ${ }^{19}$. Ceci a été sans conséquence sur les volumes sculptés parfaitement maîtrisés.

Les structures des deux œuvres sont similaires, chacune étant constituée de six pièces: la tête, le torse avec le périzonium, les deux bras et les deux jambes. Cette composition s'observe sur d'autres Christs auvergnats romans ou du début de l'art gothique (Christs d'Arlet, Auzon, Beaumont, Lavoûte-Chilhac, Lavoûte-sur-Loire, Thérondels; Christ provenant de Lavaudieu ${ }^{20}$ ) mais il en existe qui sont constitués uniquement de trois éléments ${ }^{21}$. Ce mode de fabrication peut s'expliquer en partie par la grande taille de ces deux Christs qui font environ 1,98 $\mathrm{m}$ de la tête jusqu'aux orteils. Chacune des pièces complémentaires s'adapte sur le bloc principal du torse-périzonium à l'aide d'un tenon, bloqué par une cheville sauf pour le bloc de la tête. L'imagerie par rayon X permet de visualiser le long tenon cylindrique, solidaire du bloc de la tête, qui s'insère dans le tronc. Cet assemblage est réalisé par un plan complexe, le joint dessinant un angle obtus à la base du cou avec la pointe située sur l'accroche des clavicules (fig. 3) et une courbe légère au bas de la nuque (fig. 4 et fig. 6). Cette découpe singulière et élaborée du joint assure le positionnement de l'assemblage. Comparée au joint horizontal des têtes de la plupart des Vierges assises romanes, auvergnates ${ }^{22}$, elle constitue une sophistication technique qui permet une plus grande discrétion. En ce qui concerne l'assemblage des épaules, l'emboîtement du tenon du bras dans la mortaise de l'épaule est le système d'origine mais

\footnotetext{
${ }^{19} \mathrm{Cf}$. La question de l'emplacement des évidements, développée dans la suite du texte.

${ }^{20}$ Christ dont la tête est conservée au musée du Louvre à Paris et le torse au Metropolitan Museun of Art de New York. KARGERE et al. - "Un Christ roman auvergnat...

${ }^{21}$ A l'instar du Christ crucifié de Blesle (Haute-Loire), dont seuls les bras sont rapportés sur la pièce principale (comprenant la tête, le torse, le périzonium et les jambes).

${ }^{22}$ Voir bibliographie sur les études de Vierge en majesté.
} 
nous n'avons pas d'éléments certifiant le caractère originel de la cheville de blocage. Elle est insérée par le dessus de l'épaule sur le Christ de Saint-Flour tandis qu'elle est insérée horizontalement par l'avant sur le Christ de Montsalvy. L'attache des jambes se fait sur une plateforme localisée à mi-hauteur des cuisses - plateforme préservée des évidements pratiqués à l'arrière du périzonium. Deux mortaises traversantes sont taillées (fig. 7). Les tenons des jambes viennent se caler dans cette plateforme, ils y sont bloqués par une longue cheville introduite sur le côté du périzonium (fig. 7). Le périzonium est ainsi évidé en deux endroits, en partie basse pour laisser le passage des cuisses mais en préservant le pan arrière au périzonium (visible quand le Crucifix est placé en hauteur) et en partie haute en laissant une épaisseur de bois assez mince au drapé. Parallèlement aux deux évidements réalisés à l'arrière du périzonium, une différence d'élaboration apparaît au revers du torse entre les deux Christs, celui du Christ de Saint-Flour n'étant pas évidé dans le dos à la différence de celui de Montsalvy. Chez ce dernier un large évidement débute sous les omoplates et se termine au dessus de la ceinture. Cet évidement a été pratiqué pour limiter l'apparition de fentes dans la bille, en éliminant le cœur. Il permet également d'alléger le poids de l'ensemble.
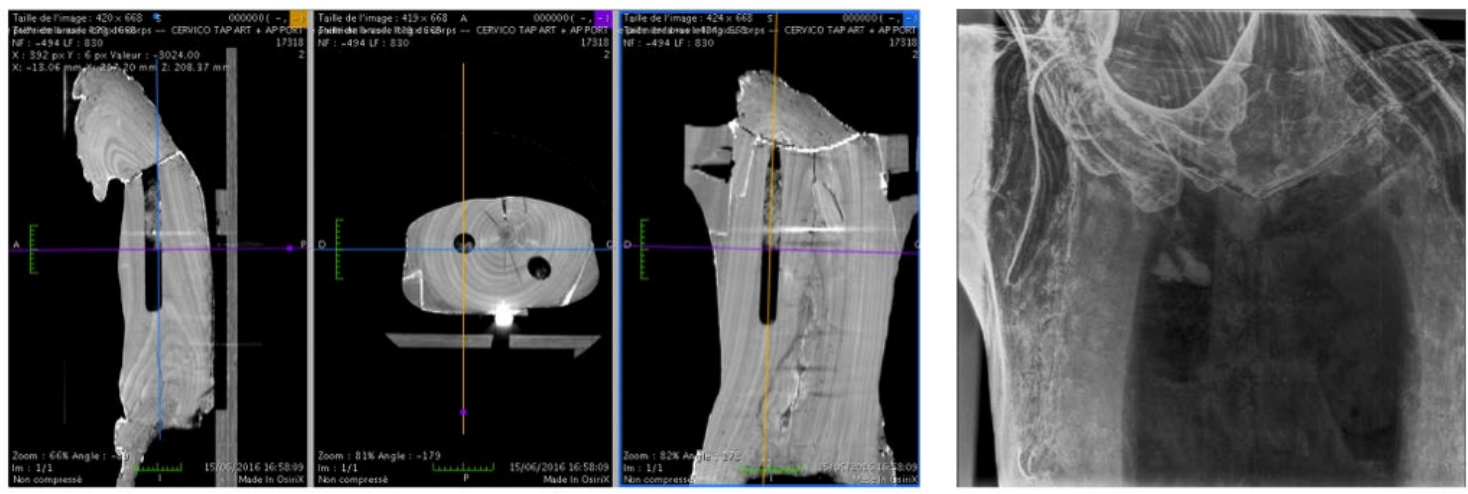

Fig. 6 - Images scanner (dans les 3 plans) du Christ de Saint-Flour montrant la cache à reliques et une partie du tenon fixant la tête au buste (à gauche); radiographie du Christ de Montsalvy montrant l'évidemment du torse, les percements de la cache à relique et du tenon fixant la tête au buste (à droite). (C Dominique Faunières et Agnès Blossier) 


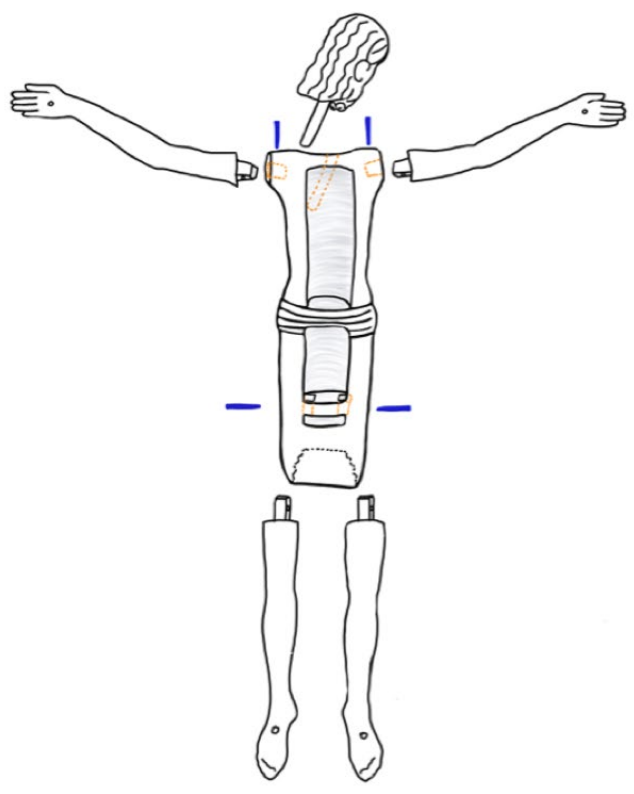

Fig. 7 - Schéma du dos du Christ de Montsalvy permettant de visualiser les différentes pièces de bois constituant la sculpture, les évidements et les modes d'assemblages. (C) Dominique Faunières et Agnès Blossier)

\section{La cache à reliques}

L'examen par imagerie médicale des deux Christs nous a permis de mettre en évidence la présence d'une cache à reliques jusque-là non décelée. Les deux Christs étudiés sont donc des œuvres renfermant des reliques dont le logement est particulièrement discret puisque aménagé à l'intérieur du torse, à partir du joint de la tête au torse, donc parfaitement invisible en surface.

Pour le Christ de Saint-Flour, on observe une masse qui pourrait correspondre à un textile roulé avec deux points d'une densité beaucoup plus importante au centre. Il pourrait s'agir d'os ou de dents. Pour le Christ de Montsalvy, la mise en évidence de textiles beige et rosé protégeant la relique a été possible grâce à une micro caméra que nous avons pu placer au niveau d'une petite communication - survenue vraisemblablement accidentellement au moment de l'élaboration - entre la cache à relique et l'évidement du torse. Ces textiles entourent probablement les reliques qui à la radiographie sous rayons $\mathrm{X}$ forment nettement deux masses opaques de petites dimensions. Ces matériaux soumis à la lecture d'un médecin radiologue ont été identifiés comme étant des dents humaines. 
On notera, par ailleurs, que lors de la conception du torse, l'évidement du dos avait été prévu alors que dans cette même zone, devaient également figurer le percement permettant l'assemblage du tenon de la tête et celui d'une loge à reliques, ce qui constitue une gageure technique (fig. 6).

\section{Les clous et l'attache à la croix}

Les quatre clous métalliques - originaux pour le Christ de Montsalvy - ont une tête bombée. Le système d'attache à l'arrière du périzonium est assez singulier. Le système est bien conservé sur le Christ de Montsalvy (fig. 8), alors qu'il ne demeure plus que des témoins des implantations des pièces métalliques pour le Christ de Saint-Flour ${ }^{23}$. Le dispositif est constitué de trois éléments: de deux barres traversant la croix et fichées dans le bois du Christ et d'une plaque clouée, appliquée à l'arrière du Christ. Les deux barres horizontales, placées en parallèle, sont de petite section coudées à leur extrémité pour se ficher dans le bois - côté Christ; elles traversent l'épaisseur de la croix pour être bloquées au revers, chacune par une goupille fendue, introduite dans un orifice percé à l'extrémité de la barre. La plaque qui est placée perpendiculairement à leur implantation est là pour renforcer le bois et éviter ainsi l'arrachement. Maintenu de la sorte, l'arrière du périzonium est parfaitement appliqué contre la croix.

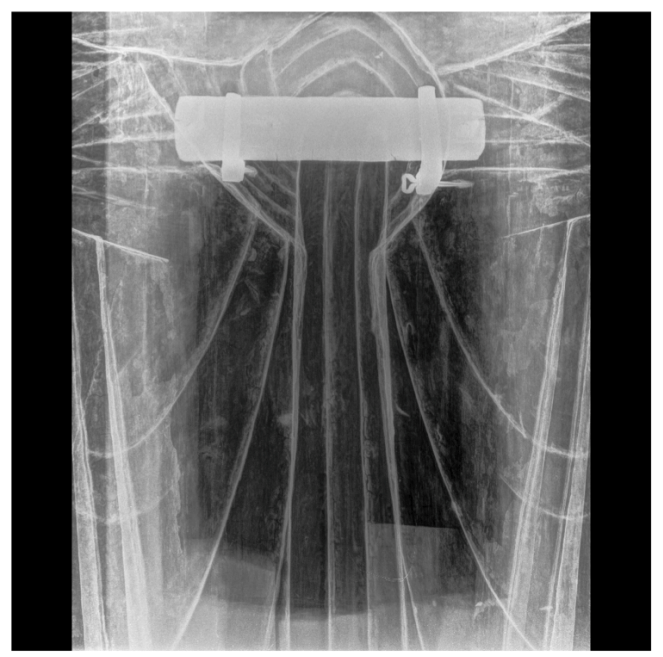

Fig. 8 - Christ de Montsalvy: radiographie du système de fixation du bassin du Christ sur la croix. (C) Dominique Faunières et Agnès Blossier)

\footnotetext{
${ }^{23}$ Rappel: le Christ de Saint-Flour est fixé sur une croix récente.
} 


\section{La croix}

La croix du Christ de Montsalvy a subi des dégradations, dont la perte de ses quatre extrémités et celles des polychromies sur la face, excepté quelques reliquats présents sous les mains. Néanmoins elle conserve, sur son revers, de nombreuses couches picturales donnant ainsi une information précieuse sur son mode de présentation dans l'édifice présentation permettant de visualiser, au moins en partie, le revers. De plus l'ancienneté de la croix et l'absence de trace d'autres dispositifs d'accrochage impliquent le caractère original de l'actuelle fixation du Christ sur sa croix, éléments rarement observés pour la période romane.

\section{La polychromie originale des Christs de Saint-Flour et de Montsalvy}

La comparaison des polychromies originales a été moins aisée à faire en raison de leur état de conservation: les carnations du Christ de Saint-Flour ont été éliminées avant leur noircissement, celles du Christ de Montsalvy, mal conservées sous les repeints, n'ont été observées qu'à l'aide d'échelles stratigraphiques. D'après nos observations, le rapprochement entre les deux Christs, est partiellement possible. Ils comportent tous deux une couche préparatoire blanche au blanc de plomb ${ }^{24}$ d'aspect huileux (ou à base d'émulsion), caractéristique des polychromies auvergnates romanes. Ils possèdent également tous deux l'indication du sang coulant des cinq plaies et un périzonium bleu comportant du lapis-lazuli. Mais là s'arrête la comparaison. Les couches de polychromie du Christ de Montsalvy sont très fines. On constate une absence de couche rose sur les carnations qui, par conséquent, devaient apparaître très blanches. L'indication du sang sur les plaies est directement peinte sur la couche préparatoire blanche. Le périzonium est composé d'une couche bleu noirâtre très sombre, posée sur la préparation. La couche colorée est extrêmement fine, de l'ordre d'un glacis. Du lapis-lazuli, très finement broyé a été identifié, il est noyé dans un liant abondant. Toujours dans le cas du Christ de

\footnotetext{
${ }^{24}$ Les analyses du laboratoire Lamoa s'appuient sur l'utilisation de la microscopie optique classique, suivie de la microanalyse des constituants - microtexture et composition - grâce à la mise en oeuvre de la microscopie électronique à balayage (MEB). Enfin, à l'aide de la sonde électronique et au détecteur de rayons $\mathrm{X}$ associé, il est possible d'analyser la composition élémentaire des constituants visibles à l'image (spectres EDX).
} 
Montsalvy aucun décor original n'a été observé ${ }^{25}$. En revanche, bien qu'altéré par un incendie, le périzonium bleu du Christ de Saint-Flour conserve des témoins substantiels d'un décor assez simple. Celui-ci est composé, d'une part, d'un semis de fleurettes stylisées en léger relief dont la particularité est leur agencement très régulier, et d'autre part, d'une large bordure ornée d'un motif mal conservé, composé de rouge et de jaune, respectivement à base de cinabre et d'orpiment (fig. 9) ${ }^{26}$. Des lignes noires soulignent les creux des plis, et des lignes jaunes rehaussent les bords du vêtement.

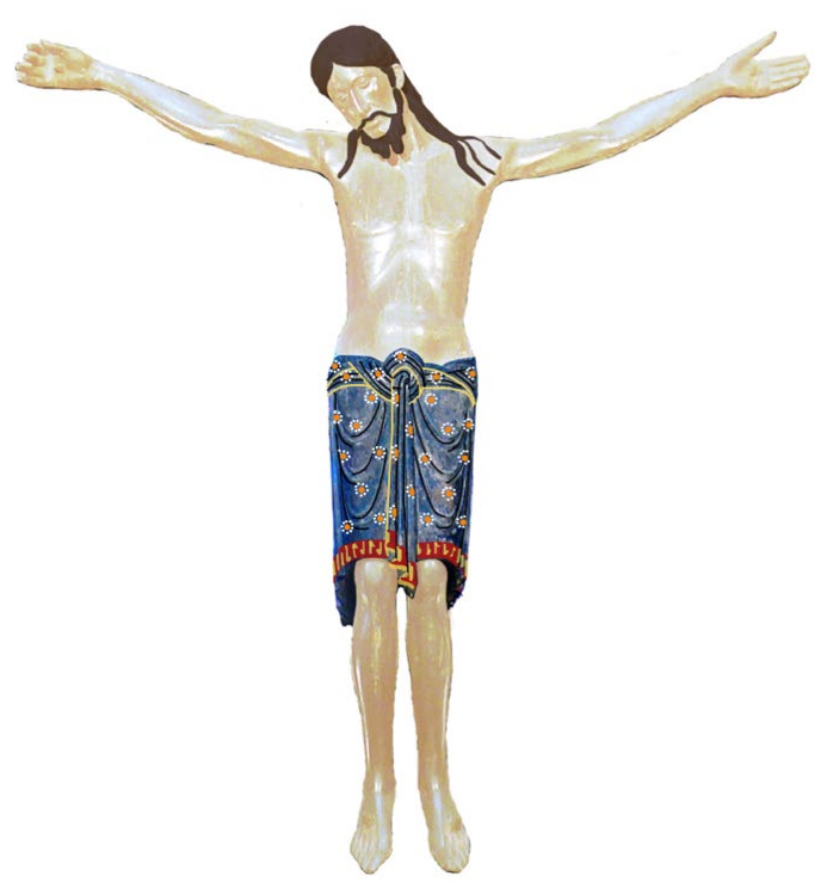

Fig. 9 - Reconstitution de la polychromie originale du Christ de Saint-Flour. (C) Dominique Faunières et Agnès Blossier)

Deux comparaisons ont pu être faites au sujet du décor de fleurettes, composé d'un centre orangé entouré de points blancs. Ce motif est présent sur un autre Christ auvergnat - celui de Blesle - dont le traitement sculptural et la structure sont relativement différents et qui a priori est plus ancien. Ce décor trouve également un écho européen, assez lointain, puisque ces mêmes fleurettes sur fond bleu ornent le Crucifix suédois de Buttle tandis

\footnotetext{
${ }^{25}$ Nous rappelons que la polychromie est à l'état de restes sous les repeints. Le périzonium conserve des vestiges de sa polychromie originale, cantonnés aux parties reculées du drapé, plus préservées. La radiographie (fig. 8) laisse deviner une sorte de petite fleur composée, opaque, qui ne peut pas être replacée dans la stratigraphie des couches picturales.

${ }^{26}$ Voir infra la polychromie du Christ de Lavoûte-Chilhac.
} 
que le reste du périzonium n'est pas comparable, ni non plus les carnations ou le style de la sculpture ${ }^{27}$ (fig. 10).

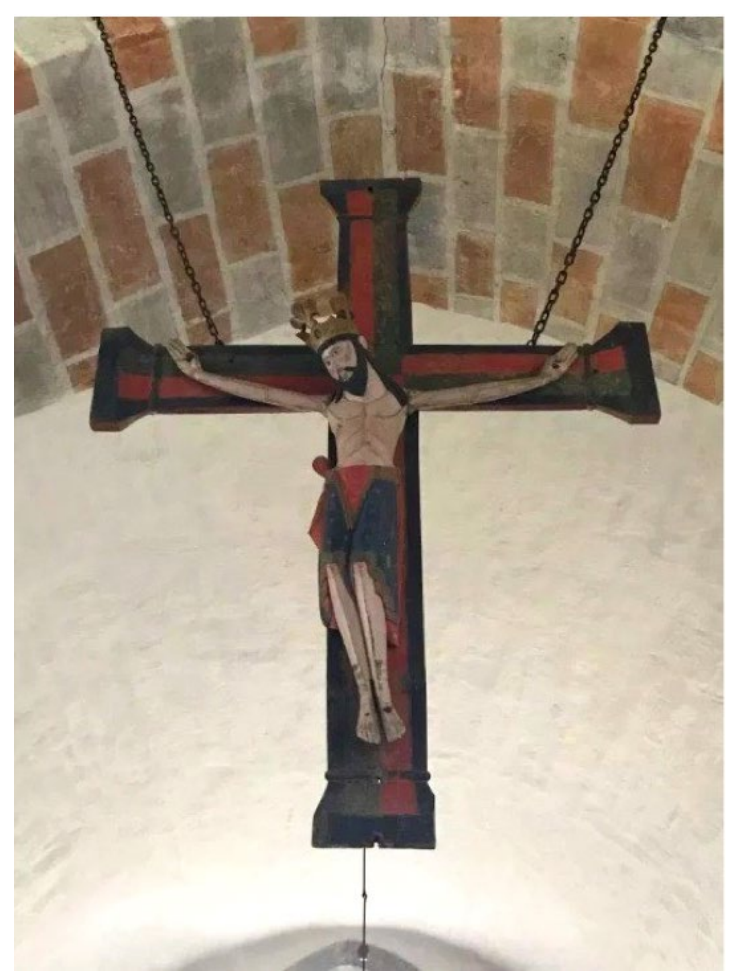

Fig. 10 - Christ de Buttle, Suède, bois polychromé, milieu du 12ème siècle. Photo internet

\section{L'apport de l'examen succinct du Christ de Lavoûte-Chilhac}

En tous points - iconographiques, stylistiques et techniques - le Christ de LavoûteChilhac apparaît similaire aux Christs précédents (fig. 11) ${ }^{28}$. Ainsi la posture, les caractéristiques anatomiques, la structure en six pièces, les détails sculptés du visage et de la chevelure, la ligne singulière du joint de la tête, le montage des jambes assemblées sur une plateforme à l'intérieur du périzonium, l'agencement du périzonium, la préservation de sa partie arrière sont autant de caractéristiques qui se retrouvent à l'identique. De plus, les nombreuses similitudes physiques et techniques remarquées ont été confortées par une série de prises de dimensions systématiques effectuées sur les trois Christs dont le tableau ci-dessous se fait l'écho.

\footnotetext{
27 TÅnGEBERG, Peter - Holzskulptur Und Altarschrein. Studien Zu Form, Material Und Technik Mittelalterliche Plastik in Schweden (1989), p. 90 and color ill no 4.

${ }^{28}$ Pour le moment, celui-ci n'a fait l'objet d'aucune étude technologique approfondie, mais nous avons pu nous rendre sur place pour faire quelques observations depuis une échelle.
} 

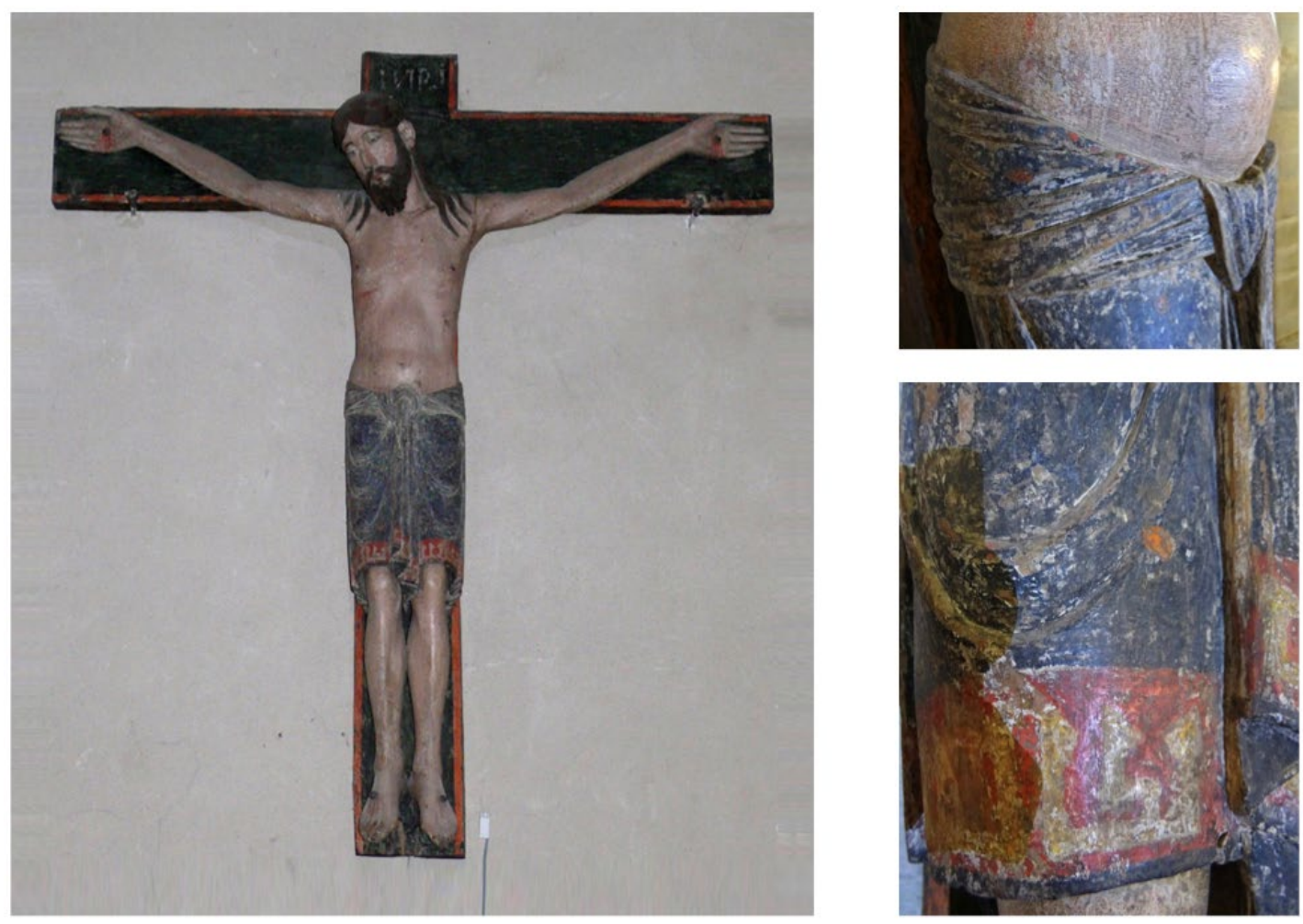

Fig. 11 - Christ de Lavoûte-Chilhac, bois polychromé, 12ème -13ème siècle, détails du périzonium. ( $(\mathcal{C}$ Dominique Faunières et Agnès Blossier)

\begin{tabular}{|c|c|c|c|}
\hline & Saint-Flour & Montsalvy & Lavoûte-Chilhac \\
\hline Hauteur & $\begin{array}{l}194 \\
\text { dimensions originelles modifiées }\end{array}$ & 198,5 & 198 \\
\hline Envergure & $\begin{array}{l}190 \\
\text { dimensions originelles modifiées }\end{array}$ & 198 & 196 \\
\hline Epaisseur maximale & 36 & 36 & 35 \\
\hline Largeur de la taille & 27,5 & 26,5 & 27,5 \\
\hline Largeur de la tête & 23,5 & 23 & Non relevé \\
\hline Épaisseur au sommet de la tête & 36 & 36 & Non relevé \\
\hline Hauteur des genoux & $\begin{array}{l}30 \\
\text { dimensions originelles modifiées }\end{array}$ & 31,5 & Non relevé \\
\hline Longueur du pied & 30 & 30 & 30 \\
\hline Épaisseur du montant de la croix & croix récente & 6,5 & 6,7 à 6,9 \\
\hline Largeur du montant de la croix & croix récente & 27,5 & 27 \\
\hline Diamètre des clous des pieds & & $22 / 24 \mathrm{~mm}$ & $24 / 25 \mathrm{~mm}$ \\
\hline
\end{tabular}

(Dimensions en $\mathrm{cm}$ ) 
Sur des parties peu remaniées aux cours des siècles, on note une variation minime de l'ordre de 5 à $10 \mathrm{~mm}$. Ces variations peuvent être expliquées par des erreurs de prise de mesures, d'une part, par des déformations du bois ou bien sûr des différences originelles, d'autre part. Il existe aussi, bien entendu, de petites différences de proportions dans les détails sculptés (longueur d'une mèche de la barbe, largeur d'un pli, etc.) mais cela paraît anecdotique et surtout très normal dans la réalisation d'une taille directe.

Comme pour Montsalvy, les extrémités de la croix de Lavoûte-Chilhac ont été affranchies nous privant aussi des dimensions et de l'allure générale du Crucifix. Les clous de la Crucifixion, à tête bombée, sont les mêmes que ceux de Montsalvy, ce qui donne à penser que la croix de Lavoûte-Chilhac serait authentique. Une étude approfondie permettrait de compléter et vérifier cette première approche qui nous semble néanmoins déjà parlante.

Bien qu'une étude des couches stratigraphiques de la polychromie n'ait jamais été faite, il existe de fortes présomptions pour qu'une partie de la polychromie apparente appartienne à l'élaboration originale ${ }^{29}$. Il est frappant d'y observer l'exacte polychromie du périzonium du Christ de Saint-Flour dans ses couleurs et son organisation (fig. 11). La préservation du décor de la large bordure rouge et jaune révèle une écriture pseudocoufique que l'on a tout lieu de penser avoir été identique pour le Christ de Saint-Flour. D'après les observations menées sur la croix de Montsalvy et celle de Lavoûte-Chilhac, il semble que les croix étaient également conçues selon un même modèle car les largeurs et épaisseurs du montant et de la traverse sont équivalentes.

\section{Des données scientifiques de datation}

La datation par le Carbone 14 du bois prélevé permet d'avancer une période, pour le Christ de Saint-Flour, allant de 1049 à 1270 avec une probabilité de 95,4\%, de 1150 à 1270 avec une probabilité de $86,3 \%$. Le resserrement de la fourchette nous semble raisonnable au regard du taux de probabilité qui reste élevé. Cela correspondrait pour la réalisation de la sculpture à une période de création de 1170-1290 en extrapolant d'une

\footnotetext{
${ }^{29}$ Son état n'est pas homogène. Lors d'une restauration on a manifestement tenté de la mettre au jour mais avec des moyens relativement sommaires.
} 
vingtaine d'années pour prendre en compte la date du cerne prélevé, la date de l'abattage de l'arbre et l'achèvement de la sculpture ${ }^{30}$. Pour Montsalvy, c'est le bois de la croix qui a été choisi pour la datation car celle-ci nous semblait ancienne et contemporaine du Christ $^{31}$, ce qui est rare en France. Curieusement on obtient une date nettement plus ancienne, à savoir une fourchette située à $95,4 \%$ de probabilité entre 915 et 1041 et une probabilité de $74,5 \%$ pour une datation située entre 960 et 1041 en extrapolant de la même manière la date de fabrication de la croix à partir de celle du bois prélevé. Ces premiers résultats confirment l'ancienneté de la croix mais soulèvent d'autres questionnements. Les intervalles de dates obtenues pour l'un et l'autre prélèvement ne se recouvrant pas, il sera peut-être nécessaire de renouveler l'opération pour la datation de la croix de Montsalvy (une contamination organique étant toujours possible) mais il sera surtout nécessaire de réaliser une datation au C14 du Christ. Initialement datées du 15ème siècle, ces deux sculptures classées sont replacées clairement dans le champ de la sculpture romane grâce à ces études.

\section{Des perspectives de recherches}

La convergence des coïncidences et des similitudes rencontrées sur ces trois Christs est confondante $^{32}$. Cela nous amène à proposer qu'au delà de la suggestion d'un même artiste ou atelier qui nous paraît évidente, les Christs étudiés (Saint-Flour, Montsalvy) ou celui simplement examiné relativement succinctement (Lavoûte-Chilhac) forment une série homogène. Deux hypothèses peuvent être formulées. Soit cette série appartient à un seul et même programme qui pourrait émaner du même commanditaire, dignitaire ou ordre religieux. Soit cette production correspond à la réplication à l'unité et à la demande d'un modèle primitif. Dans les deux cas, en acceptant ce travail, l'atelier s'engageait à tenir compte d'une considérable contrainte, celle de reproduction fidèle.

Ces hypothèses engendrent des questionnements quant à l'existence ou à la définition d'un modèle préalable ou d'une œuvre de référence, à la volonté particulière d'une

\footnotetext{
${ }^{30}$ Le prélèvement a été effectué en périphérie de la bille mais ne comportait pas l'aubier du tronc.

${ }^{31}$ Lors de la commande de l'étude, un seul prélèvement pour C14 avait été programmé. Les observations du Christ ne permettant aucun doute sur son ancienneté, nous avons choisi de faire un prélèvement sur la croix.

${ }^{32}$ Les différences de mise en œuvre, observées, peuvent a priori traduire des contraintes spécifiques ou de nouvelles solutions techniques apportées au fil de la réalisation.
} 
réplication "à l'identique", mais également quant aux raisons et aux moyens qui ont permis que l'artiste (ou son atelier) accepte cette contrainte. La réalisation pourrait avoir été simultanée ou plus étalée dans le temps. Cette production de Christs monumentaux aurait pu être commandée pour plusieurs édifices ayant un lien entres eux, voire appartenant éventuellement au même ordre religieux.

Le nombre initial pourrait s'étendre au delà des trois sculptures citées précédemment. En effet, l'observation récente de deux autres œuvres auvergnates, a priori composites, justifie à notre sens de nouveaux rapprochements (fig. 12). Certaines parties constitutives de ces deux sculptures, semblent appartenir à ce groupe. Il s'agit de la tête du Christ conservé dans le trésor de la cathédrale de Clermont-Ferrand (Puy-de-Dôme) (fig. 13) et de la tête et du torse ${ }^{33}$ du Christ actuellement conservé dans le réfectoire de l'abbaye de Lavaudieu (Haute-Loire) (fig. 13) ${ }^{34}$. Au delà des remaniements, suite à des altérations qui devraient bien sûr être répertoriées précisément, les caractéristiques de la sculpture et les quelques mesures prises sont là aussi comparables.

\footnotetext{
${ }^{33}$ Le périzonium est remanié.

${ }^{34}$ Dans le cas de Clermont-Ferrand, le reste du corps est de facture différente; dans le second cas il n'y a pas de correspondance entre le haut et le bas du corps. Le Christ du Trésor a été vu à hauteur mais à une certaine distance, le Christ du réfectoire de Lavaudieu a pu être examiné de plus près et assez rapidement. Nous remercions sincèrement MM Pierre-Olivier Benech et Pierre Taillefer ainsi que Christiane BessonBenoit, Daniel Bailly, Pierre-Yves Le Pogam et Lucretia Kargère pour leur aide lors de ces dernières visites.
} 

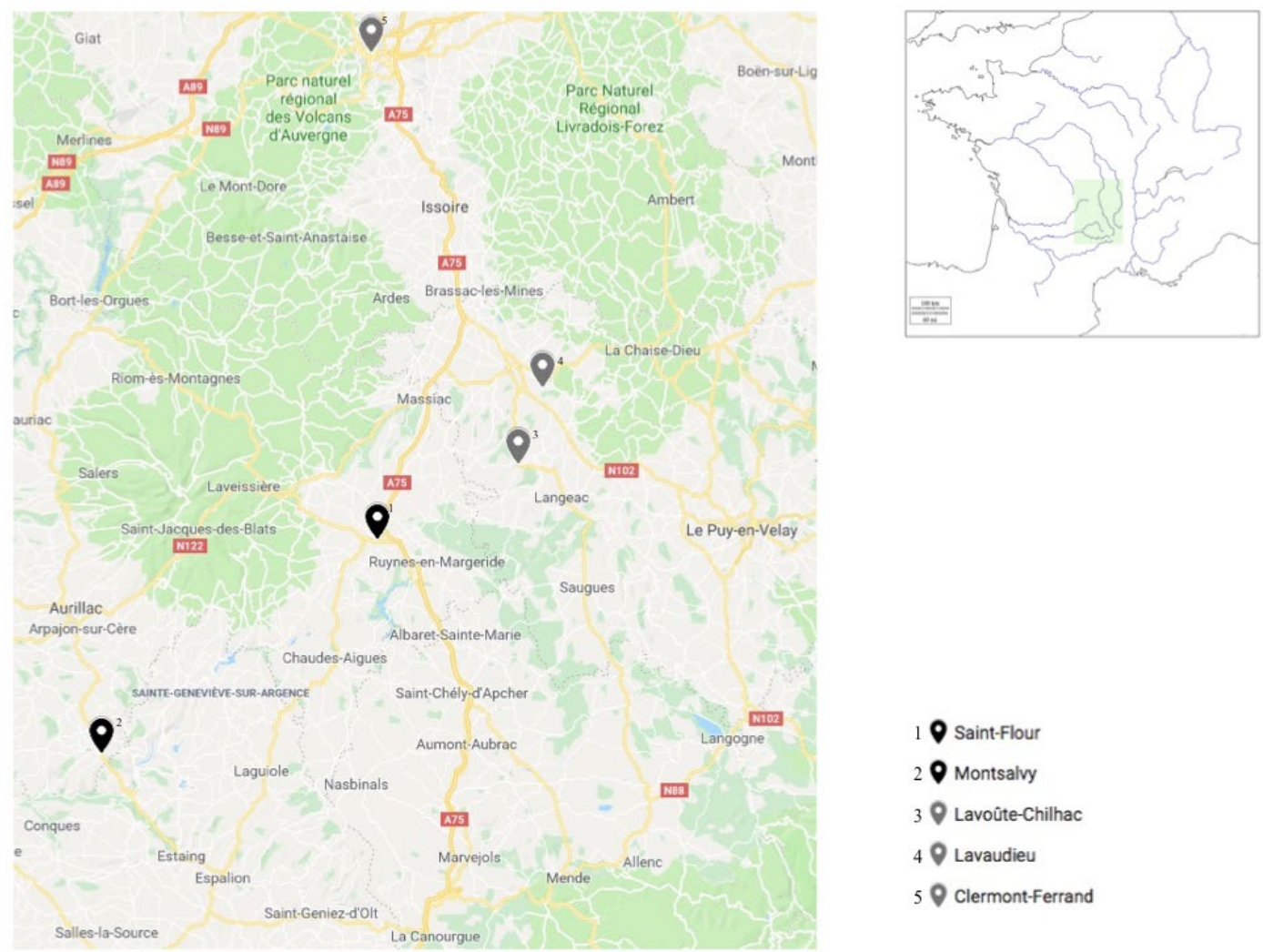

Fig. 12 - Carte de la région Auvergne, localisation des Christs mentionnés.
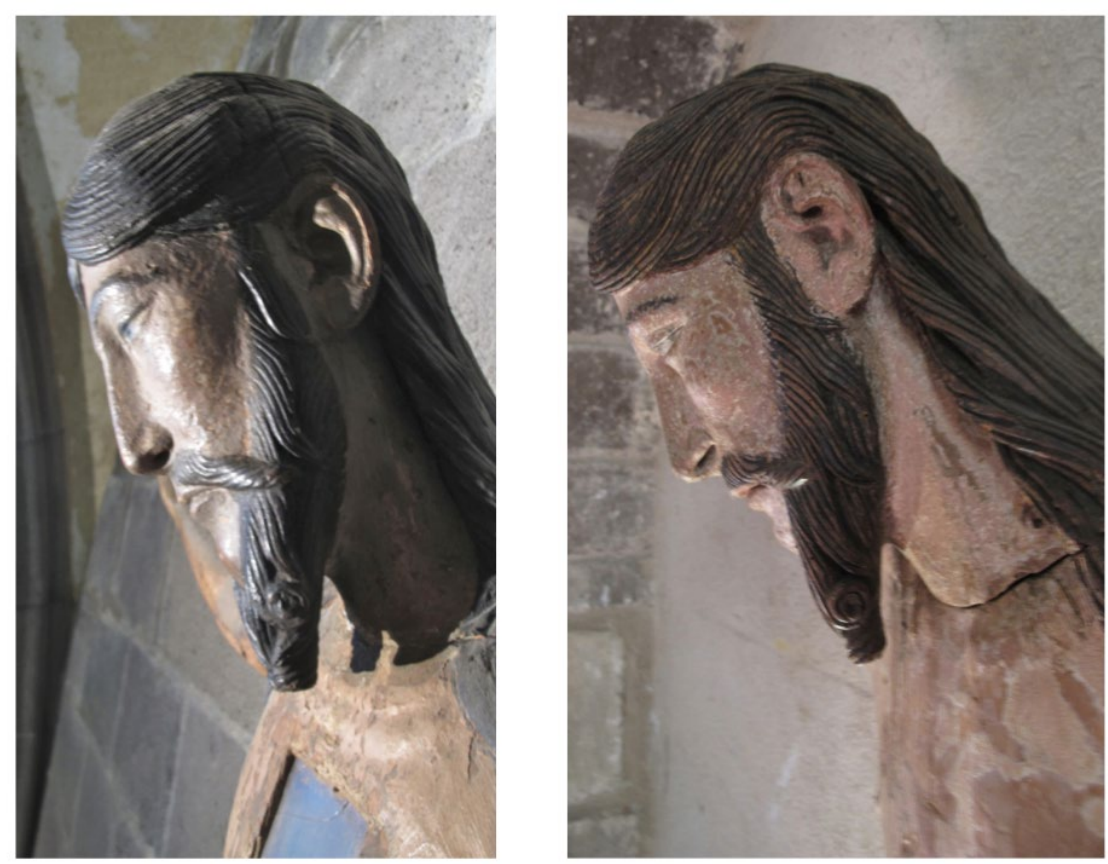

Fig. 13 - Christ conservé dans le trésor de la cathédrale de Clermont-Ferrand, bois polychromé, 12ème-13ème siècle (à gauche) et Christ conservé dans le réfectoire de l'abbaye de Lavaudieu, bois polychromé, 12ème -13ème siècle (à droite). (C Dominique Faunières et Agnès Blossier) 
Concernant l'élaboration des sculptures et l'obtention de dimensions quasiment identiques, il paraît évident que l'atelier était organisé de manière à pouvoir répéter (reporter aisément) un modèle ou une œuvre de référence. Abordaient-ils une seule sculpture à la fois ou plusieurs? En cas de commande multiple, les torses étaient-ils sculptés d'un côté, tandis que les têtes étaient dégrossies d'un autre, avant d'être parfaitement ajustées? S'agit-il du travail d'un seul sculpteur ou de plusieurs mains au sein du même atelier? Pouvait-il exister une sorte de répartition des tâches? Comment reportaient-ils les dimensions? Existait-il des gabarits, des contre-formes?

Lors de travaux de recherches récents la problématique de copies originales à l'époque médiévale a été soulevée, principalement pour le 15ème siècle ${ }^{35}$. Que le phénomène puisse déjà exister à la période romane pour des objets en bois, donc datables par l'analyse au Carbone 14 et authentifiables aussi par les techniques de polychromies connues, ajoute une donnée supplémentaire, sans doute essentielle à la question.

Les croix étaient-elles toutes de mêmes dimensions et élaborées selon un même modèle tout comme les accessoires de fixations en alliage ferreux forgé (fixations à la croix et clous)? D'après nos observations la phase sculpture et la phase polychromie pourraient être imbriquées et donc réalisées dans un même atelier, comme le suggère la présence de polychromie originale sur le dessus des cuisses et le revers des genoux - inaccessibles après le montage des jambes. La polychromie de la croix était réalisée au moins en partie avant que la sculpture ne soit en place. Les polychromies originales du périzonium du Christ de Saint-Flour et du Christ de Lavoûte-Chilhac présentent une similitude de traitement (fond bleu lapis-lazuli parsemé de fleurettes rouge orangé et blanches) qui pourrait avoir à nouveau la même origine, celle d'une commande très spécifique. Ce motif simple est assez commun dans l'art médiéval, puisqu'on le trouve dans l'enluminure ${ }^{36}$ et en peinture ${ }^{37}$. Nous relevons en revanche l'élaboration différente et plus succincte de la

\footnotetext{
${ }^{35}$ Voir en bibliographie les parutions sur le sujet, transmises aimablement par Pierre-Yves Le Pogam, conservateur en chef au musée du Louvre, qui nous a informé de ce contexte plus large de répliques. Nous le remercions chaleureusement pour sa relecture attentive et productive.

${ }^{36}$ TROYES, Bibliothèque municipale, Ms. 60, 1180, illustré en Italie du Nord, $\mathrm{f}^{\circ}$ 8-10-85-98-213.

${ }^{37}$ Euvres catalanes du 12ème siècle (conservées au musée national d'art de Catalogne à Barcelone): devant d'autel de La Seu d'Urgell, devant d'autel d'Ix, peinture murale de l'abside de Santa Maria de Taüll (manteau de la Vierge). Peinture murale de l'église Sant Marti de La Cortinada (Andorre, fin du 12ème siècle). En France, peinture murale de l'église de Brinay (Cher, 12ème siècle).
} 
polychromie du Christ de Montsalvy. Dans ce dernier cas, le parti pris pouvait-il correspondre à une exécution rapide (et à moindre coût) dans la perspective d'un événement important, en attendant une élaboration à venir plus aboutie et conforme à la demande initiale? À moins que cette distinction de niveau dans l'exécution des polychromies ne suive pas l'exigence de réplication observée pour les formes sculptées.

Les études techniques, en permettant le décrochage des œuvres parfois présentées très en hauteur et par conséquence assez éloignées du regard, ont abouti à des observations pointues. Un rapprochement physique de ces Christs serait également une bonne occasion d'évaluer les hypothèses avancées. Dans le cas présent, l'interprétation menée interroge la création romane au delà de la simple question de la réalisation matérielle. Il semble nécessaire de confronter nos hypothèses à la connaissance du contexte historique et religieux de la région auvergnate. La poursuite de ces recherches au groupe plus élargi des Crucifix auvergnats sera probablement l'occasion de comprendre plus précisément le contexte des commandes et la création à l'époque romane.

\section{Lucretia Kargère:}

\section{Facture en série des sculptures d'Auvergne}

La compréhension du mode d'exécution des sculptures et le savoir de leur(s) créateur(s) restent un sujet peu compris, pouvant être largement amélioré par l'analyse directe et l'examen scientifique des œuvres. Le choix des essences de bois, leur utilisation, mode de taille et outils, emploi de joints, et finition, forment un ensemble de données utiles à la classification des centres de production. Les sculptures étudiées en Auvergne, Vierges et Christ en Croix, semblent tous suivre des modèles, et former des groupes homogènes, non seulement de par leur aspect stylistique, mais également de par leur méthode de taille, construction, et finition picturale. Ce schéma cependant reste assez général, sans un travail sur la facture en séries de ces sculptures, car cette homogénéité peut s'atténuer après l'examen scientifique. Doit-on regarder ces sculptures d'une manière linéaire dans l'évolution d'un atelier, d'une formule simple à plus complexe? Ou bien plutôt, de la reproduction d'un même modèle, peut-être renommé, à divers niveaux de qualité? 
Les Vierges dites appartenir à l'atelier du Maitre "Morgan" notamment sont le projet d'une étude prolongée cherchant à démontrer les similarités ou disparités d'un nombre de sculptures de facture similaire ${ }^{38}$. Ont été associées à cet atelier dont l'œuvre principale est la Vierge Morgan du Metropolitan Museum, les sculptures de Montvianeix (Puy-deDôme) au Musée du Cloisters, celle de Notre-Dame d'Usson (Puy-de-Dôme) dans la collection du musée d'art Roger-Quilliot, Notre-Dame de Claviers à Moussages (Cantal), Notre-Dame d'Aubusson à Aubusson d'Auvergne (Puy-de-Dôme), et d'Heume-l'Eglise (Puy-de-Dôme) (fig.14). Notre-Dame de la Bonne Mort aujourd'hui dans la cathédrale de Clermont-Ferrand ressemble fortement à ce groupe, bien que la plupart des surfaces aient été restaurées au début du 19ème siècle, redorée, noircie, aux chaussures et trône certainement récents ${ }^{39}$. Sans rayon $X$, le reste d'une possible âme médiévale est difficile à délimiter. Très similaires de composition notamment dans l'asymétrie des mains de la Vierge, la frontalité de l'enfant, le registre des drapées, les détails physionomiques de mains aux longs doigts, ou d'oreilles fort charnues (fig.15), les sculptures ne sont pas identiques, contrairement aux Christs ci-dessus présentés. Leur spécificité a été vite perçue lors d'une exposition au Cloisters de New York, ou les Vierges de Montvianeix et Morgan ont été présentées côte à côte (fig.16) ${ }^{40}$. Ces Vierges ne sont pas de la même échelle et envergure, peut-être pour accommoder des niches ou autels de différentes tailles selon les églises. Leur finition notamment dans la finesse de la taille des cheveux, ou bien dans l'amplitude des plissés délimitée dès le sommet de la tête, diffère (fig.17). De même, les surfaces peintes, bien que très similaires, sont des variations sur un thème, plutôt que des copies conformes. Par exemple, un coussin décoré de quatre-feuilles verts sur fond bleu pâle pour la Vierge de Morgan se transforme en ce même dessin en rose et blanc pour celle de Montvianeix. Ou encore des ornements géométriques en étain trouvés sur les manteaux de la Vierge et de l'Enfant de Montvianeix, sont déplacés sur les bordures des manches et rebords du trône pour celle de Morgan. Cependant, le mode de construction et technique de polychromie de ces sculptures est pratiquement identique montrant un savoir-faire commun.

\footnotetext{
${ }^{38}$ KARGÈRE et RIZZO - "Twelfth-century French polychrome sculpture...”, pp. 38-72; BLOSSIER, Agnès - “Etude et rapport d'intervention”, Vierge à l'Enfant, Heume-l'Eglise”, Juillet 2012.

${ }^{39}$ Observation Lucretia Kargère, Octobre 2018.

${ }^{40}$ Exposition Heavenly Bodies: Fashion and the Catholic Imagination, May 10- October 8, 2018.
} 


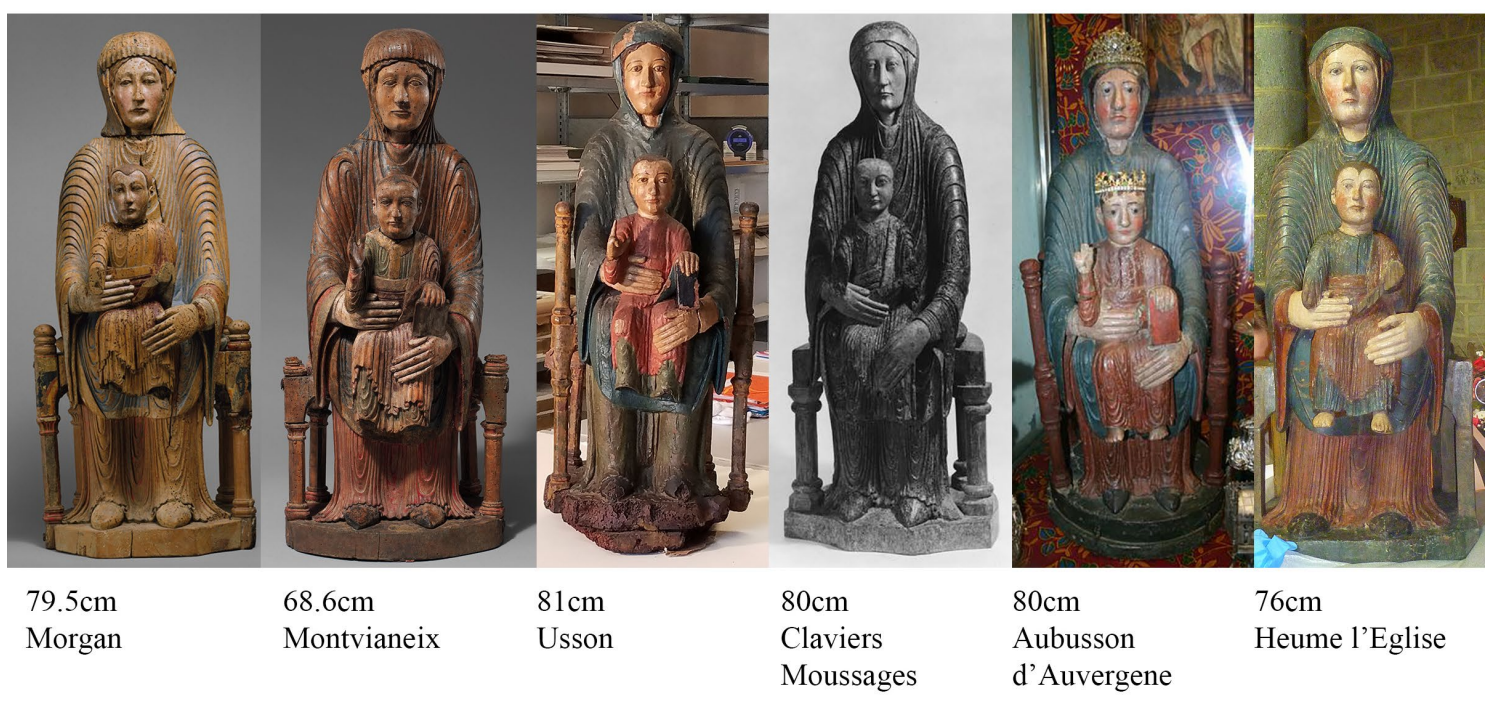

Fig. 14 - Image groupée des Vierges en majesté, bois polychromé, 12ème siècle, région d'Auvergne: Vierge en majesté connue sous le nom de Morgan, 79.5 x 31.7 x $29.2 \mathrm{~cm}$, bois polychromé, The Metropolitan Museum of Art, Gift of J. Pierpont Morgan (16.32.194) (C) Department of Objects Conservation, The Metropolitan Museum of Art, Lucretia Kargère); Vierge en majesté dite provenir de Montvianeix, $68.6 \times 28.6 \times 27.9 \mathrm{~cm}$, bois polychromé, The Metropolitan Museum of Art, The Cloisters Collection 1967 (67.153) (C Department of Objects Conservation, The Metropolitan Museum of Art, Lucretia Kargère); Vierge en majesté dite Notre Dame d'Usson, Musée d'Art Roger Quilliot, 2002.1.1, (C Lucretia Kargère); Vierge en majesté de l'Eglise de Notre-Dame-de Claviers (C Emmanuelli), Médiathèque de l'architecture et du patrimoine; Vierge en majesté de l'Eglise d'Aubusson d'Auvergne (C) Monuments historiques), 1993; Vierge en majesté d'Heume-l'Eglise. (C) Lucretia Kargère et Agnès Blossier)
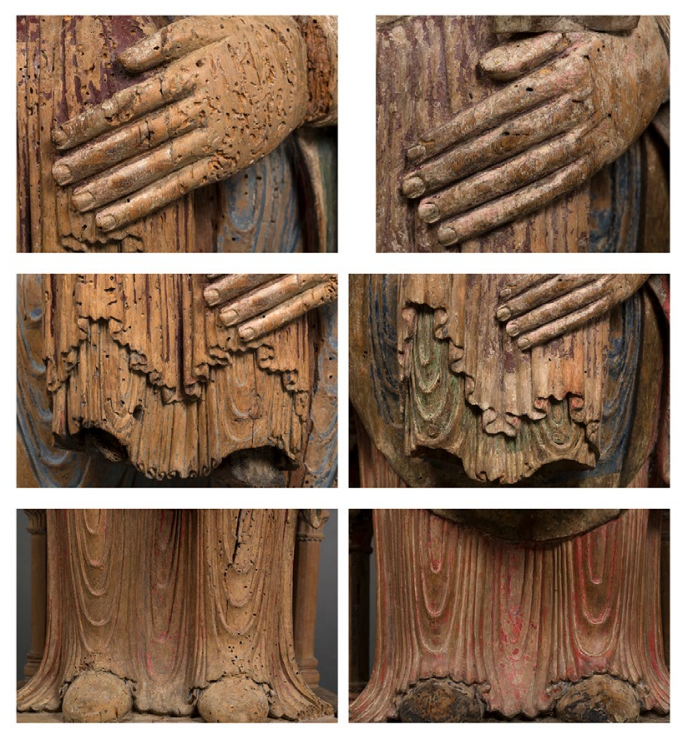

Fig.15 - Détails des Vierges de Morgan (à gauche) et Montvianeix (à droite). (C Department of Objects Conservation, The Metropolitan Museum of Art, Lucretia Kargère) 


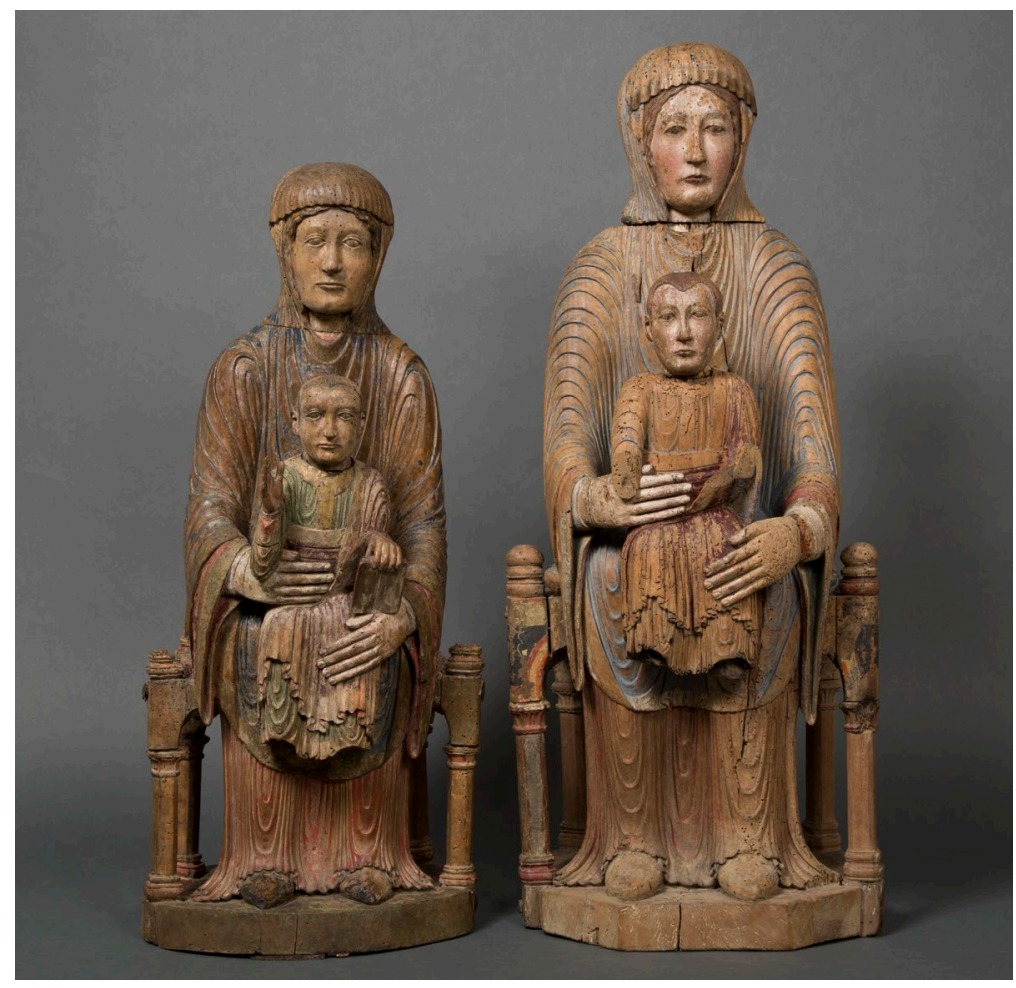

Fig.16 - Vierge en majesté de Montvianeix (à gauche) et de Morgan (à droite).

(C Department of Objects Conservation, The Metropolitan Museum of Art, Lucretia Kargère)

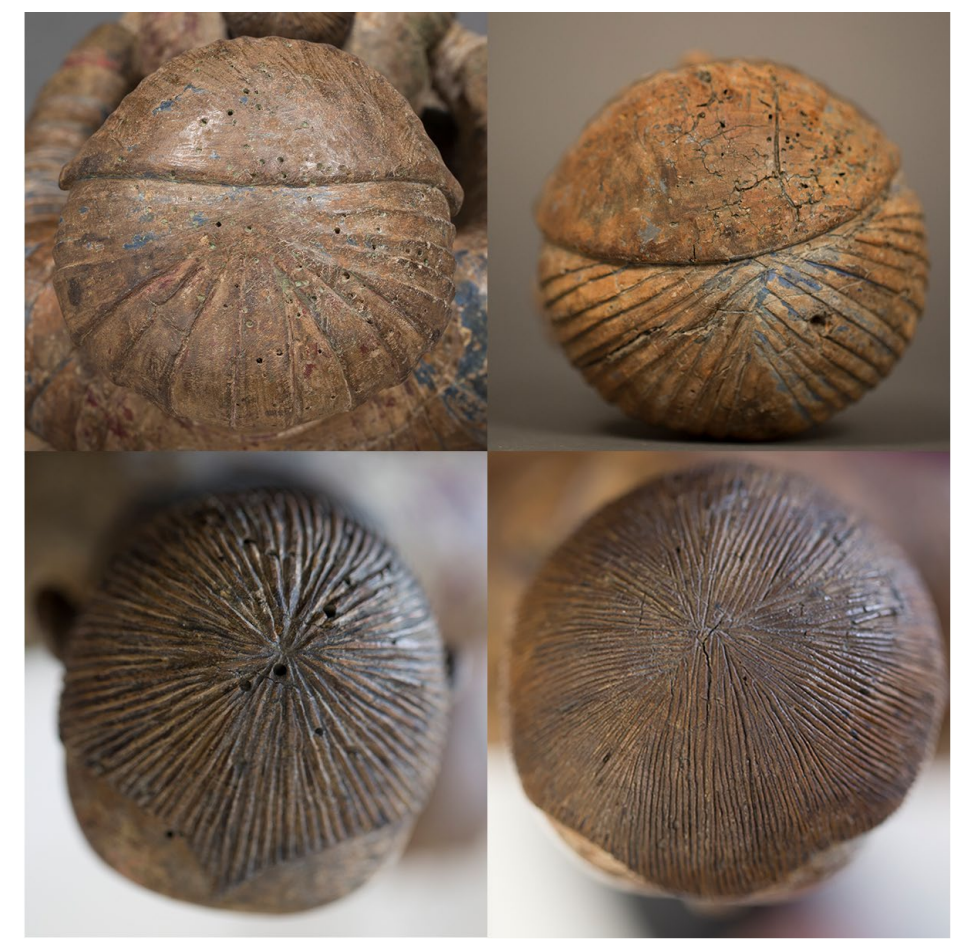

Fig.17 - Détails du dessus des têtes de la Vierge et de l'Enfant: Vierge en majesté de Montvianeix (à gauche) et de Morgan (à droite).

(C) Department of Objects Conservation, The Metropolitan Museum of Art, Lucretia Kargère) 
Comme il a été déjà démontré, les Vierges sont assemblées au moins d'une douzaine de pièces de bois, avec têtes séparées du tronc principal, et colonnes du trône encastrées dans ce même bloc. Montvianeix et Morgan sont toutes deux sculptées en noyer, mais il semble que ce bois soit couramment utilisé dans la région. Le corps principal de la sculpture n'est pas évidé, et le cour du bois se trouve au quasi centre de la composition. Il en est de même pour la polychromie dont la palette de pigments, colorants et mode d'application, liant inclus, est identique pour les Vierges de Montvianeix et Morgan. Notamment, le bleu au lapis des manteaux des Vierges sur une sous-couche grise (veneda), les glacis de laque rouge, l'emploi de reliefs en étain, les carnations à l'huile sur une couche translucide d'huile de lin, également notée, bien que sans analyse, sur la Vierge d'Heumel'Église et celle d'Usson ${ }^{41}$.

\section{Une meilleure compréhension du contexte créatif}

L'anonymat domine dans l'art roman, et beaucoup d'inconnus subsistent quant à la formation technique et au mode de travail des sculpteurs et peintres. Une plus ample documentation a été fournie pour l'Italie, certaines régions de France, et la Catalogne ${ }^{42}$.

Les sculpteurs sur bois étaient-ils des artistes-moines spécialisés? Ou bien faisaient-ils partie des chantiers de sculpture sur pierre joignant des centres urbains, ou plutôt des artistes ou artisans polyvalents travaillant également l'ivoire, ou même l'orfèvrerie? La similitude d'élaboration des Christs présentés ci-dessus, aurait-elle été observée pour d'autres de ces matériaux? L'évolution du partage des tâches dans la production artistique a certainement progressé au cours du Moyen Age. Des rapprochements entre sculptures sur bois et pierre ont déjà été recensées, notamment la comparaison de la Vierge à l'Enfant d'Autun du Musée du Cloisters aux sculptures bourguignonnes en pierre de l'atelier de Gislebertus ${ }^{43}$; ou bien encore du Christ en bois polychromé de Moissac au tympan de la

\footnotetext{
${ }^{41}$ L'étude technique de Notre Dame d'Usson est en cours avec un partenariat entre le musée d'art RogerQuilliot et l'auteur au Metropolitan Museum de New York. Une couche translucide (non analysée) a été également notée sur les Christs étudiés ci-dessus, cependant elle a été trouvée pour les carnations autant que pour le perizonium. Cette technique demande donc à être étudiée sur un champ plus large de sculptures. ${ }^{42}$ CASTIÑEIRAS, Manuel - "Artiste-Clericus ou artiste-laïque? Apprentissage et Curriculum Vitae du peintre en Catalogne et en Toscane”, Les Cahiers de Saint-Michel de Cuxa XLIII (2012), pp.15-30.

${ }^{43}$ FORSYTH, Ilene - The Throne of Wisdom ... pp. 188-189.
} 
même église ${ }^{44}$; et encore de la Vierge à l'Enfant du Fogg Museum de Harvard, ou de celle de Saint-Denis issue de Saint-Martin-des-Champs, aux sculptures de la façade de Senlis ${ }^{45}$. Les décors d'étain en relief trouvés sur les Vierges de Morgan et Montvianeix, ou bien même, dans un champ plus large, ceux des portes en bois de la cathédrale du Puy-enVelay (1143-1155), sont comparables au travail du métal au champlevé ${ }^{46}$. Les peintres embellissant les sculptures se rapprochaient forcément du savoir de l'artiste-moine ou d'un moine clericus dédié à l'art de l'enluminure, suivant des recettes préservées et développées dans cet environnement. Une collaboration proche entre sculpteur et peintre est clairement décelable sur les groupes de Christs où la peinture des jambes est rendue inaccessible après leur montage. S'agissait-il d'un artiste polyvalent, ou bien d'un atelier traitant des séquences de tâches bien ordonnées?

\section{Méthode d'approche pour les années à venir}

Ce n'est qu'avec un travail de recensement et d'étude systématique de ces sculptures que l'on pourra fournir une meilleure classification et mieux comprendre les échanges artistiques régionaux, et influences au-delà des frontières.

Au vu de ces éléments, la DRAC ARA met en réseau les études menées par les communes de son territoire, et cherche à diffuser et échanger à l'international sur ces recherches. Dès 2016, afin de projeter efficacement cette diffusion, elle recense chaque année les œuvres susceptibles d'être étudiées selon un cahier des charges concerté et normé. En 2018, la DRAC a recruté par le biais d'un marché à bon de commande un groupement de restaurateurs, pour quatre années, afin de permettre un dialogue suivi sur ces problématiques. Les œuvres en musée sont étudiées en parallèle, stimulées par la progression des connaissances des œuvres sur le territoire d'Auvergne. De par le nombre de questions restant en suspens quant à la datation des sculptures, et les nouvelles données apportées par les études récentes soit par le biais du Carbone 14 ou bien par l'étude des couches de polychromie, le corpus des sculptures en bois polychromé d'Auvergne est un projet en évolution.

\footnotetext{
44 SAUERLÄNDER, W. - "Zum dem romanischen Kruzifix von Moissac”, Intuition und Kunstwissenschaft. Festschrift für Hanns Swarzenski (1973), pp. 303-317.

${ }^{45}$ FORSYTH, Ilene - The Throne of Wisdom..., pp. 197-199.

${ }^{46}$ CAHN, Walter - "The Romanesque wooden doors of Auvergne", 1974; BARRAL I ALTET, Xavier Contre l'Art Roman? Essai sur un passe réinventé. Paris: Ed. Fayard, 2006, p.166.
} 


\section{BIBLIOGRAPHICAL REFERENCES:}

\section{Sources non publiées}

BLOSSIER, Agnès - Étude et rapport d'intervention, Vierge d'Heume-l'Église, POTTE, Marie-Blanche (dir.), DRAC ARA, 2012.

FAUNIÈRES, Dominique; BLOSSIER, Agnès - Rapport d'étude Christ crucifié dit Christ noir, Saint-Flour, Dir. M.-B. Potte, DRAC ARA, 2017.

FAUNIÈRES, Dominique; BLOSSIER, Agnès - Rapport d'étude, Christ crucifié, Montsalvy, Dir. P.-O. Benech, DRAC ARA, 2018.

FAUNIÈRES, Dominique; BLOSSIER, Agnès - Rapport d'étude, Christ de Blesle, POTTE, Marie-Blanche (dir.), DRAC ARA, 2015.

TROYES, Bibliothèque municipale, Ms. 60, 1180, illustré en Italie du Nord, fo 8-10-8598-213.

\section{Sources publiées}

ALEIL, Pierre François - "Les grands Crucifix de bois en Haute-Auvergne ". Revue de la Haute-Auvergne 38 (1963), pp. 219-236.

BARRAL I ALTET, Xavier - Contre l'Art Roman? Essai sur un passé réinventé. Paris: Ed. Fayard, 2006.

BERNÉ Damien - "Le Christ de Crucifixion de la collégiale d'Herment (Puy-de-Dôme), Une redécouverte exceptionnelle au musée de Cluny". Fines, Archéologie et Histoire en Combraille 6 (2011), pp. 17-25.

BERTONI CREN, Nadia - La sculpture sur bois polychrome des $11^{e}$ et $12^{e}$ siècles en Bourgogne. Dijon: Université de Bourgogne, 2013, PhD Thesis. 
BOEHM, Barbara - "Un buste reliquaire du 12ème siècle récemment découvert à SaintFlour ". Patrimoine en Haute-Auvergne 23/2 (2011), pp. 39-50.

BOUSQUET, Jacques - "Sur quelques Christ romans du Rouergue et le problème des ‘christs Auvergnats'”. Actes du Congres d'études de Rodez (1975), pp. 333-359.

BRÉHIER, Louis - “Les origines de l'architecture romane en Auvergne: l'œuvre des chapitres et des monastères”. Revue Mabillon (Jan 1923), pp. 8-25.

BRÉHIER, Louis - "Notes sur les Statues de Vierges Romanes, Notre Dame de Montvianeix et la question des Vierges Noires". Revue d'Auvergne 47 (1933) pp.193198.

CAHN, Walter - The Romanesque wooden doors of Auvergne, New York: University Press for the College Art Association of America, 1974.

CARRIÈRE, Adeline - "Les grands crucifix romans de bois de l'Auvergne et de ses marges". Recherche en histoire de l'art (2008), pp.7-28.

CASCIO, Agnès; DESCHAMPS-TAN, Stéphanie; LE POGAM, Pierre-Yves - "La restauration du Christ Courajod: la luminosité d'une polychromie romane retrouvée". Technè 39 (2014), pp. 52-59.

CASSAGNES-BROUQUET, Sophie - Vierges Noires. Rodez: ed. du Rouergue, 2000.

CASTIÑEIRAS, Manuel - “Artiste-Clericus ou artiste-laïque? Apprentissage et Curriculum Vitae du peintre en Catalogne et en Toscane". Les Cahiers de Saint-Michel de Cuxa XLIII (2012), pp. 15-30.

DELMAS, Claire - “Le Crucifix Roman de Salles-la-Source: Nouveaux éléments concernant les grands Christs en bois du Massif Central". Revue de la Haute-Auvergne 9 (1990), pp. 49-54. 
DESCHAMPS-TAN, Stéphanie; FAUNIÈRES, Dominique; LE POGAM, Pierre-Yves; PAGÈS-CAMAGNA, Sandrine - "La Vierge et l'Enfant en majesté, musée du Louvre". Technè 39 (2014), pp. 66-72.

FACHECHI, Grazia Maria - "Forma quantitate et qualitate diligentissime denotata la scultura lignea medievale tra spazio e funzione". Annali della pontificia insigne accademia di belle arti lettere dei virtuosi al Pantheon (2015), pp. 377-388.

FORSYTH, Ilene H - The Throne of Wisdom: Wood Sculptures of the Madonna in Romanesque France. Princeton: Princeton University Press, 1972.

FOUILHERON, Joël - "Du bon Dieu de Saint-Flour au Christ noir de la Cathédrale". Revue de la Haute-Auvergne 39 (1965), pp. 490-496.

FOUILHERON, Joël - La Cathédrale de Saint-Pierre de Saint-Flour. Paris: Nouvelles éditions latines, 1966.

GABORIT, Jean-René et FAUNIÈRES, Dominique. Une Vierge en majesté. Paris: Musée du Louvre Editions, 2009.

GIRELLI, Francesca - "Local traditions and itinerant artists?: The Crucifixes of Auvergne and Wooden Sculpture in France during the Late Romanesque Period". Convivium, Exchanges and Interactions in the Arts of Medieval Europe, Byzantium, and the Mediterranean 4/2 (2017), pp. 53-70.

KARGÈRE, Lucretia, Adriana RIZZO - “Twelfth-century French polychrome sculpture in The Metropolitan Museum of Art: materials and techniques". Metropolitan Museum Studies in Art, Science and Technology 1, New York: The Metropolitan Museum of Art and Yale University Press (2010), pp. 39-72.

KARGÈRE, Lucretia; LE POGAM, Pierre-Yves; LEVY-HINSTIN, Juliette; PINGAUD, Nathalie - "Un Christ roman auvergnat retrouve son unité grâce à l'étude de la polychromie". TECHNE 39 (2014), pp. 60-65. 
KARGÈRE, Lucretia - “The Cloisters Lavaudieu Torso of Christ: technical examination of a Romanesque crucifix from Auvergne ". Striking Images: Christ on the Cross \& The Emergence of Medieval Monumental Sculpture. ed. Gerhard Lutz, Marietta Cambareri and Shirin Fozi, Brepols/Harvey Miller, 2019.

KARGÈRE, Lucretia - "The Montvianeix Madonna: materials and techniques in $12^{\text {th }}$ century Auvergne". Proceedings ICOM Committee for Conservation $13^{\text {th }}$ Triennial Meeting Rio de Janeiro 2 (2002), pp. 507-512.

LEROY, Hélène; DEBAISIEUX, Francis - Vierges romanes, portraits croisés. Beaumont: Edition Debaisieux, 2009.

LITTLE, Charles - "Romanesque sculpture in North American collections: The Metropolitan Museum of Art; Part VI: Auvergne, Burgundy, Central France, Meuse Valley, Germany". Gesta 26/2 (1987).

LITTLE, Charles T. - “Glorious replication. Medieval sculpture from the Ile-de France”. Hommage à Renate Eikelmann (to be published).

MOULIER, Pierre - Christs romans d'Auvergne. Patrimoine en Haute Auvergne 35, 2018.

PHALIP, Bruno - L'art Roman en Auvergne. Nonette: Ed. Créer, 2003.

PLAGNIEUX, Philippe - 'D'une chapelle de la Vierge l'autre: l'exemple du prieuré clunisien de Saint-Martin-des-Champs à Paris". Bulletin du centre d'étude médiévales d'Auxerre, BUCEMA, Hors-série 6 (2013), pp.1-13.

ROCHEMONTEIX, Adolphe de - Les églises romanes de Haute-Auvergne. Paris: Picard, 1902.

SAUERLÄNDER, W. - “Zum dem romanischen Kruzifix von Moissac”. Intuition und Kunstwissenschaft. Festschrift für Hanns Swarzenski (1973), pp. 303.317. 
Etudes menées sur les sculptures d'Auvergne en bois polychromé - Marie-Blanche Potte, et al.

TÅNGEBERG, Peter - Holzskulpturen und Altarschreine, Studien zu Form, Material und Technik Mittelalterliche Plastik in Schweden. München: Edition Callwey, 1986.

Bibliographie complémentaire relative aux copies dans la sculpture médiévale

BERTRAND, Sylvaine - “Trois Vierges à l'Enfant du $15^{\mathrm{e}}$ siècle aux environs de Beaune. Leur type, leur style et leur place dans la production bourguignonne du XV siècle". Centre bois d'études historiques 76 (1996), pp. 11-22.

GABORIT, Jean-René - "Le problème de la copie dans la sculpture médiévale". Tables de travail. Séminaire de recherche sur la conservation-restauration, [accessed 2 April 2019]. Available at http://tablesdetravail.hypotheses.org/108.

LAPAIRE, Claude - "Une statue bourguignonne de saint Thibaut et l'usage des modèles, répliques et réductions dans les ateliers de sculpture du XV ${ }^{\mathrm{e}}$ siècle”. Genava 31 (1983), pp. 27-33. 


\section{COMO CITAR ESTE ARTIGO}

\section{Referência electrónica:}

POTTE, Marie-Blanche; FAUNIÈRES, Dominique; BLOSSIER, Agnès; KARGÈRE, Lucretia - "Etudes menées sur les sculptures d'Auvergne en bois polychromé".

Medievalista 26 (Julho-Dezembro 2019). [Em linha] [Consultado dd.mm.aaaa].

Disponível em http://www2.fcsh.unl.pt/iem/medievalista/MEDIEVALISTA26/potte-etal2608.html

ISSN 1646-740X.

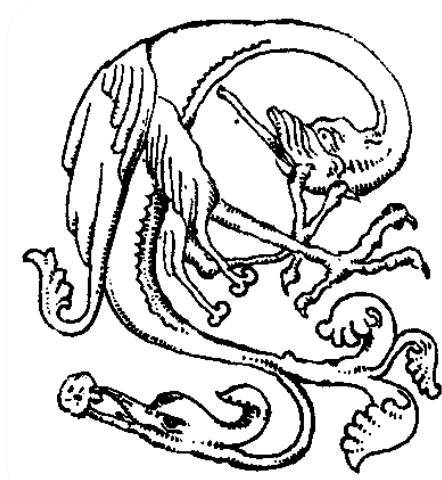

